\title{
The Gaia-ESO Survey: properties of newly discovered Li-rich giants ${ }^{\star}$
}

R. Smiljanic ${ }^{1}$, E. Franciosini2 ${ }^{2}$, A. Bragaglia ${ }^{3}$, G. Tautvaišiené $\dot{4}^{4}$, X. Fu ${ }^{3,5}$, E. Pancino ${ }^{2,6}$, V. Adibekyan ${ }^{7}$, S. G. Sousa ${ }^{7}$, S. Randich ${ }^{2}$, J. Montalbán ${ }^{8}$, L. Pasquini ${ }^{9}$, L. Magrini ${ }^{2}$, A. Drazdauskas ${ }^{4}$, R. A. García ${ }^{10,11}$, S. Mathur ${ }^{12,13,14}$, B. Mosser ${ }^{15}$, C. Régulo ${ }^{12,13}$, R. de Assis Peralta ${ }^{15}$, S. Hekker ${ }^{16,17}$, D. Feuillet $^{18}$, M. Valentini ${ }^{19}$, T. Morel ${ }^{20}$, S. Martell ${ }^{21}$, G. Gilmore ${ }^{22}$, S. Feltzing ${ }^{23}$, A. Vallenari ${ }^{24}$, T. Bensby ${ }^{23}$, A. J. Korn ${ }^{25}$, A. C. Lanzafame ${ }^{26}$,

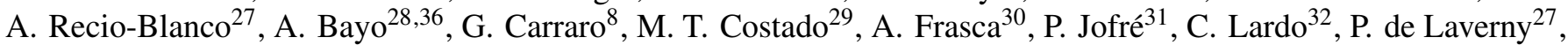
K. Lind ${ }^{18,25}$, T. Masseron ${ }^{12,13}$, L. Monaco ${ }^{33}$, L. Morbidelli ${ }^{2}$, L. Prisinzano ${ }^{34}$, L. Sbordone ${ }^{35}$, and S. Zaggia ${ }^{24}$

(Affiliations can be found after the references)

Received 15 March 2018 / Accepted 17 May 2018

\begin{abstract}
Aims. We report 20 new lithium-rich giants discovered within the Gaia-ESO Survey, including the first Li-rich giant with an evolutionary stage confirmed by CoRoT (Convection, Rotation and planetary Transits) data. We present a detailed overview of the properties of these 20 stars. Methods. Atmospheric parameters and abundances were derived in model atmosphere analyses using medium-resolution GIRAFFE or highresolution UVES (Ultraviolet and Visual Echelle Spectrograph) spectra. These results are part of the fifth internal data release of the Gaia-ESO Survey. The Li abundances were corrected for non-local thermodynamical equilibrium effects. Other stellar properties were investigated for additional peculiarities (the core of strong lines for signs of magnetic activity, infrared magnitudes, rotational velocities, chemical abundances, and Galactic velocities). We used Gaia DR2 parallaxes to estimate distances and luminosities.

Results. The giants have $A(\mathrm{Li})>2.2$ dex. The majority of them (14 of 20 stars) are in the CoRoT fields. Four giants are located in the field of three open clusters, but are not members. Two giants were observed in fields towards the Galactic bulge, but likely lie in the inner disc. One of the bulge field giants is super Li-rich with $A(\mathrm{Li})=4.0 \mathrm{dex}$.

Conclusions. We identified one giant with infrared excess at $22 \mu \mathrm{m}$. Two other giants, with large v sin $i$, might be Li-rich because of planet engulfment. Another giant is found to be barium enhanced and thus could have accreted material from a former asymptotic giant branch companion. Otherwise, in addition to the Li enrichment, the evolutionary stages are the only other connection between these new Li-rich giants. The CoRoT data confirm that one Li-rich giant is at the core-He burning stage. The other giants are concentrated in close proximity to the red giant branch luminosity bump, the core-He burning stages, or the early-asymptotic giant branch. This is very clear from the Gaia-based luminosities of the Li-rich giants. This is also seen when the CoRoT Li-rich giants are compared to a larger sample of 2252 giants observed in the CoRoT fields by the Gaia-ESO Survey, which are distributed throughout the red giant branch in the $T_{\text {eff }}-\log g$ diagram. These observations show that the evolutionary stage is a major factor for the $\mathrm{Li}$ enrichment in giants. Other processes, such as planet accretion, contribute at a smaller scale.
\end{abstract}

Key words. stars: abundances - stars: evolution - stars: late-type

\section{Introduction}

Although more than three decades have past since the discovery of the first Li-rich giant (Wallerstein \& Sneden 1982), the origin of such objects remains without a clear explanation (see Lyubimkov 2016, for a review). According to standard stellar evolution models, the surface Li abundances of low-mass red giants after the first dredge-up should lie below $A(\mathrm{Li}) \sim 1.50 \mathrm{dex}$ (e.g. Lagarde et al. 2012). However, about 1-2\% of the known giants have been found to be richer in $\mathrm{Li}$ than this (see, e.g. Casey et al. 2016; Kirby et al. 2016).

Different scenarios have been proposed to explain their highLi abundances. These scenarios can be broadly divided into those requiring internal fresh $\mathrm{Li}$ production, and those postulating external pollution by material rich in Li. A few additional processes have been proposed to explain some specific cases within the zoo of Li-rich giants, such as the red giant branch

* Based on observations collected at the European Organisation for Astronomical Research in the Southern Hemisphere under ESO programmes 188.B-3002 and 193.B-0936 (The Gaia-ESO Public Spectroscopic Survey).
(RGB) phase transition discussed in Cassisi et al. (2016) and the extra-mixing inhibition discussed in Smiljanic et al. (2016).

Internal Li production likely takes place by the mechanism proposed by Cameron \& Fowler (1971). In this mechanism, the unstable isotope ${ }^{7} \mathrm{Be}$ is produced in the stellar interior by the reaction ${ }^{3} \mathrm{He}(\alpha, \gamma)^{7} \mathrm{Be}$, which is followed by the decay ${ }^{7} \mathrm{Be}\left(e^{-} v\right)^{7} \mathrm{Li}$, resulting in freshly synthesised ${ }^{7} \mathrm{Li}$. The Cameron \& Fowler mechanism, however, was introduced to explain observations of $\mathrm{Li}$ in asymptotic giant branch (AGB) stars. In AGB stars, the convective layer enters the H-burning shell providing the means to bring the fresh $\mathrm{Li}$ to the surface (see, e.g. Scalo et al. 1975; Palmerini et al. 2011). In firstascent red giants, however, the introduction of an extra mixing mechanism is required to bring the fresh $\mathrm{Li}$ to the surface before it is destroyed in a reaction with protons of the medium. The physical mechanism responsible for this fast deep mixing is still unknown (Sackmann \& Boothroyd 1999; Charbonnel \& Balachandran 2000; Palacios et al. 2001).

The engulfment of planets and/or planetesimals is often the preferred scenario of external pollution to explain $\mathrm{Li}^{-}$ rich giants (see, e.g. Siess \& Livio 1999; Aguilera-Gómez et al. 
2016; Delgado Mena et al. 2016; Reddy \& Lambert 2016). The location in the Herztsprung-Russel (HR) diagram of Li-rich giants previously discovered within the Gaia-ESO Survey seemed to be consistent with those of giants that engulfed close-in giant planets before evolving up the RGB (Casey et al. 2016). In this scenario, the Li enhancement should be accompanied by enhancement of other light elements, such as ${ }^{6} \mathrm{Li}$ and Be. Enhancement in these elements, however, has never been detected in any of the Li-rich giants investigated so far (de Medeiros et al. 1997; Castilho et al. 1999; Melo et al. 2005; Takeda \& Tajitsu 2017; Adamów et al. 2018). Moreover, at least in red giants, the complexity of the evolutionary mixing events affecting $\mathrm{Li}$ and other elements has so far precluded the discovery of clear abundance signatures related to planet engulfment (see, e.g., Carlberg et al. 2016b,a). This kind of joint $\mathrm{Li}$ and Be enhancement seems to have been detected in only one main-sequence star so far: in the open cluster NGC 6633, as reported by Ashwell et al. (2005).

It has been suggested that the Li enhancement in giants might be connected to a phase of enhanced mass loss (de la Reza et al. 1996, 2015). In this context, the star KIC 4937011, located in the field of the open cluster NGC 6819, is very interesting. The asteroseismic analysis of stars in NGC 6819 by Stello et al. (2011) classified KIC 4937011 as a non-member of the cluster. The oscillation pattern of KIC 4937011 was found to be different from that of other stars at similar position in the colour-magnitude diagram (CMD). This star was later found to be Li-rich by Anthony-Twarog et al. (2013). They suggested that the asteroseismic mismatch could be related to the process that caused the Li enrichment. Carlberg et al. (2015) investigated KIC 4937011 in detail and summarised all the evidence supporting cluster membership (such as radial velocity and overall chemical composition). More recently, Handberg et al. (2017) suggested that KIC 4937011 is indeed a member of NGC 6819 , but that it experienced very high mass loss during its evolution (it has now $0.7 M_{\odot}$ compared to $1.6 M_{\odot}$ of other red giants in the cluster).

Asteroseismology, thus, seems to offer the means to uncover new information about Li-rich giants. Four other Li-rich giants observed with Kepler (Borucki et al. 2010) have been reported (Silva Aguirre et al. 2014; Jofré et al. 2015; Bharat Kumar et al. 2018). Nine Li-rich giants observed with CoRoT (Convection, Rotation and planetary Transits, Baglin et al. 2006; Auvergne et al. 2009) have been discovered with Gaia-ESO data and were reported in Casey et al. (2016).

This work is the second Gaia-ESO paper on the subject of Li-rich giants. Here, we report the discovery of 20 new Li-rich giants, 14 of which are in the CoRoT fields. These new Li-rich giants were discovered in new Gaia-ESO observations that were not available in the data release used in Casey et al. (2016). With the new stars, there are now $40 \mathrm{Li}$-rich giants identified using Gaia-ESO data. Our goal is to discuss the properties of these new Li-rich giants and discuss which clues they provide about the origin of the Li enrichment. In particular, this offers the opportunity to revisit the conclusions of Casey et al. (2016) using the more recent reanalysis of Gaia-ESO data available in the Survey's fifth internal data release (iDR5). We also take advantage of new PARSEC isochrones (Fu et al. 2018) and parallaxes of the recent second data release (DR2) of Gaia (Gaia Collaboration 2016, 2018; Lindegren et al. 2018) to revisit the discussion about the position of the Li-rich giants in the HR diagram.

This paper is organised as follows. In Sect. 2 we give a brief description of the Gaia-ESO Survey (Gilmore et al. 2012; Randich \& Gilmore 2013) data and analysis. In Sect. 3 we present the properties of the 20 newly discovered Li-rich giants. In Sect. 4 we use CoRoT data and Gaia-based luminosities to update the discussion about the origin of the Li enrichment in the Li-rich giants discovered by the Gaia-ESO Survey. Finally, Sect. 5 summarises our findings.

\section{Data and analysis}

The spectra used here have been obtained with the FLAMES (Fibre Large Array Multi Element Spectrograph, Pasquini et al. 2002) instrument at the European Southern Observatory's (ESO) Very Large Telescope (VLT) in Cerro Paranal, Chile. FLAMES was used to feed both the GIRAFFE medium-resolution $(R \sim 20000)$ and the UVES (Ultraviolet and Visual Echelle Spectrograph, Dekker et al. 2000) high-resolution $(R \sim 47000)$ spectrographs.

Basic information on the newly discovered Li-rich giants is given in Table 1. All stars were observed with GIRAFFE, except for the two giants towards the bulge and the CoRoT target with CNAME 19265193+0044004, which were observed with UVES. The Li line at $6708 \AA$ falls within the GIRAFFE setup HR15N ( $\lambda \lambda 647-679 \mathrm{~nm})$. In Gaia-ESO, this GIRAFFE setup is only used to observe calibrators (such as the CoRoT stars) and stars in open clusters. All other stars in Milky Way fields are observed only with HR10 and/or HR21 and therefore the determination of $\mathrm{Li}$ abundances is not possible for them. UVES, on the other hand, is used to observe mainly FG-type dwarfs in the solar neighbourhood, giants in the field of open clusters, and towards the Bulge, or (bright) calibrators, such as some CoRoT giants. Because of these details of how the stars are observed, Li-rich giants have been discovered with Gaia-ESO data only in these three types of fields (open clusters, CoRoT fields, or towards the Bulge).

An overview of the GIRAFFE data reduction can be found in Jackson et al. (2015). The UVES data reduction is described in Sacco et al. (2014).

The atmospheric parameters, effective temperature $\left(T_{\text {eff }}\right)$, surface gravity $(\log g)$, metallicity $([\mathrm{Fe} / \mathrm{H}])$, and microturbulence $(\xi)$, projected rotational velocities $(v \sin i)$, and the chemical abundances are part of iDR5. The values of atmospheric parameters are given in Table 2. The spectra were analysed using the Gaia-ESO multiple pipelines strategy. For the case of stars in the field of young clusters, which can also contain pre-main sequence stars, the spectrum analysis was described in Lanzafame et al. (2015). The analysis of UVES spectra of other FGK-type stars was described in Smiljanic et al. (2014), and an updated discussion will be presented in Casey et al. (in prep.). The analysis of GIRAFFE spectra of other FGKtype stars was briefly described in Recio-Blanco et al. (2014). The results obtained in the different analyses are homogenised using the comprehensive set of stellar calibrators described in Pancino et al. (2017). The homogenisation process will be described in Hourihane et al. (in prep.). A brief description of the Gaia-ESO atomic and molecular data is given in Heiter et al. (2015). The analysis made use of the MARCS model atmospheres (Gustafsson et al. 2008).

The Li abundances were determined from the $6708 \AA$ line assuming local thermodynamical equilibrium (LTE). A combination of methods including spectrum synthesis, measurement of equivalent widths, and curve of growths was used. The line list includes the hyperfine structure and isotopic splitting of the $\mathrm{Li}$ line and the Fe $\mathrm{I}$ blend at $6707.4 \AA$. We selected from the iDR5 catalogue giants $(\log g \leq 3.5 \mathrm{dex})$ with $T_{\text {eff }}$ between 4000 and $5000 \mathrm{~K}$, and with detected Li abundances, not upper limits, where $A(L i) \geq 2.0$ dex (in LTE). Example spectra of three Li-rich giants are shown in Fig. 1. 
Table 1. Observational data of the new-Li rich giants.

\begin{tabular}{|c|c|c|c|c|c|c|c|}
\hline CNAME & Field & 2MASS ID & $\begin{array}{c}\text { RA } \\
\text { h:m:s (J2000) }\end{array}$ & $\begin{array}{c}\text { Dec } \\
\mathrm{d}: \mathrm{m}: \mathrm{s}(\mathrm{J} 2000)\end{array}$ & $\begin{array}{c}V \\
\text { mag }\end{array}$ & $\begin{array}{c}\mathrm{RV} \\
\mathrm{km} \mathrm{s}^{-1}\end{array}$ & $S / N$ \\
\hline $08405643-5308309$ & IC 2391 & 08405643-5308309 & $8: 40: 56.43$ & $-53: 08: 30.90$ & 14.48 & +55.0 & 35 \\
\hline 17522490-2927512 & Rup 134 & $17522490-2927512$ & $17: 52: 24.90$ & $-29: 27: 51.20$ & $15.16^{1}$ & +81.6 & 47 \\
\hline $17531013-2932063$ & Rup 134 & $17531013-2932063$ & $17: 53: 10.13$ & $-29: 32: 06.30$ & $14.10^{1}$ & -25.8 & 99 \\
\hline $18181062-3246291$ & Bulge & $18181061-3246290$ & $18: 18: 10.62$ & $-32: 46: 29.10$ & $11.68^{1}$ & +39.5 & 184 \\
\hline $18182698-3242584$ & Bulge & $18182697-3242584$ & $18: 18: 26.98$ & $-32: 42: 58.40$ & $12.67^{1}$ & +27.4 & 135 \\
\hline $18265248+0627259$ & NGC 6633 & $18265248+0627259$ & $18: 26: 52.48$ & $+06: 27: 25.90$ & 14.45 & +32.7 & 51 \\
\hline $19223053+0138518$ & Corot & $19223052+0138520$ & $19: 22: 30.53$ & $+01: 38: 51.80$ & 13.03 & -23.8 & 47 \\
\hline $19251759+0053140$ & Corot & $19251759+0053141$ & $19: 25: 17.59$ & $+00: 53: 14.00$ & 14.55 & +88.7 & 39 \\
\hline $1134+0051569$ & Corot & $19261134+0051569$ & 19:26: & $+00: 51: 56.90$ & 15.02 & +29.5 & 26 \\
\hline $19263808+0054441$ & Corot & $19263807+0054441$ & $19: 26: 38.08$ & $+00: 54: 44.10$ & 13.28 & -57.8 & 66 \\
\hline $19264134+0137595$ & Corot & $19264133+0137595$ & $19: 26: 41.34$ & $+01: 37: 59.50$ & 14.25 & +44.0 & 50 \\
\hline 19264917-0027469 & Corot & $19264917-0027469$ & $19: 26: 49.17$ & $-00: 27: 46.90$ & $15.83^{2}$ & +77.9 & 20 \\
\hline+0149070 & Corot & $19265013+0149071$ & 19:26: & $+01: 49: 07.00$ & 15.86 & -48.3 & 31 \\
\hline $19265193+0044004$ & Corot & $19265195+0044004$ & $19: 26: 51.93$ & $+00: 44: 00.40$ & 13.09 & -12.9 & 116 \\
\hline $19270600+0134446$ & Corot & $19270600+0134446$ & $19: 27: 06.00$ & $+01: 34: 44.60$ & 14.87 & +28.5 & 42 \\
\hline $19270815+0017461$ & Corot & $19270815+0017461$ & $19: 27: 08.15$ & $+00: 17: 46.10$ & $15.26^{1}$ & +31.3 & 24 \\
\hline $19273856+0024149$ & Corot & $19273856+0024149$ & $19: 27: 38.56$ & $+00: 24: 14.90$ & 15.37 & +18.3 & 22 \\
\hline $19274706+0023447$ & Corot & $19274706+0023448$ & $19: 27: 47.05$ & $+00: 23: 44.70$ & $14.78^{2}$ & +46.5 & 27 \\
\hline $19280508+0100139$ & Corot & $19280507+0100139$ & $19: 28: 05.08$ & $+01: 00: 13.90$ & 15.25 & +75.0 & 44 \\
\hline $19283226+0033072$ & Corot & $19283226+0033072$ & $19: 28: 32.26$ & $+00: 33: 07.20$ & 14.77 & +49.8 & 32 \\
\hline
\end{tabular}

Notes. The $V$ magnitudes are from APASS (Henden et al. 2015) unless otherwise noted: ${ }^{(1)}$ The Guide Star Catalog, Version 2.3.2 (GSC2.3) (STScI, 2006), and ${ }^{(2)}$ the NOMAD catalogue (Zacharias et al. 2004). For the determination of radial velocities and values of signal-to-noise per pixel from the GIRAFFE spectra see Jackson et al. (2015). For the UVES spectra, the determination of these values is described in Sacco et al. (2014).

Table 2. Atmospheric parameters, lithium abundances, and rotational velocities for the newly discovered Li-rich giants.

\begin{tabular}{lccccccccccrr}
\hline \hline CNAME & $\begin{array}{c}T_{\text {eff }} \\
(\mathrm{K})\end{array}$ & $\begin{array}{c}\sigma \\
(\mathrm{K})\end{array}$ & $\log g$ & $\sigma$ & {$[\mathrm{Fe} / \mathrm{H}]$} & $\sigma$ & $\begin{array}{c}\xi \\
\mathrm{km} \mathrm{s}^{-1}\end{array}$ & $\begin{array}{r}\sigma \\
\mathrm{km} \mathrm{s}^{-1}\end{array}$ & $\begin{array}{r}A(\mathrm{Li}) \\
(\mathrm{LTE})\end{array}$ & $\begin{array}{r}\sigma \\
(\mathrm{Li})\end{array}$ & $\begin{array}{r}\mathrm{v} \sin i \\
\mathrm{~km} \mathrm{~s}^{-1}\end{array}$ \\
\hline $08405643-5308309$ & 4486 & 142 & 2.54 & 0.15 & -0.12 & 0.12 & - & - & 2.64 & 0.18 & 2.60 & $\leq 7.0$ \\
$17522490-2927512$ & 4644 & 188 & 2.80 & 0.14 & +0.18 & 0.16 & - & - & 2.32 & 0.25 & 2.42 & $\leq 7.0$ \\
$17531013-2932063$ & 4557 & 169 & 2.72 & 0.18 & +0.27 & 0.13 & - & - & 2.12 & 0.10 & 2.30 & $\leq 7.0$ \\
$18181062-3246291$ & 4558 & 57 & 2.27 & 0.11 & -0.03 & 0.06 & 1.54 & 0.13 & 2.15 & 0.06 & 2.30 & $2.5 \pm 3.4$ \\
$18182698-3242584$ & 4425 & 57 & 2.33 & 0.11 & +0.10 & 0.13 & 1.53 & 0.29 & 4.12 & 0.06 & 4.04 & $6.0 \pm 4.6$ \\
$18265248+0627259$ & 4982 & 192 & 2.88 & 0.23 & -0.08 & 0.23 & - & - & 2.74 & 0.10 & 2.69 & $37.1 \pm 2.0$ \\
$19223053+0138518$ & 4579 & 42 & 2.49 & 0.09 & +0.26 & 0.23 & - & - & 2.06 & 0.03 & 2.27 & $\leq 7.0$ \\
$19251759+0053140$ & 4621 & 38 & 2.78 & 0.09 & +0.36 & 0.20 & - & - & 2.03 & 0.04 & 2.24 & $\leq 7.0$ \\
$19261134+0051569$ & 4745 & 39 & 2.47 & 0.09 & -0.53 & 0.22 & - & - & 3.60 & - & 3.25 & $7.5 \pm 1.7$ \\
$19263808+0054441$ & 4655 & 31 & 2.82 & 0.09 & +0.38 & 0.12 & - & - & 2.09 & 0.10 & 2.29 & $12.0 \pm 3.0$ \\
$19264134+0137595$ & 4645 & 36 & 2.56 & 0.09 & +0.28 & 0.20 & - & - & 3.60 & - & 3.45 & $\leq 7.0$ \\
$19264917-0027469$ & 4458 & 46 & 2.19 & 0.10 & -0.39 & 0.26 & - & - & 3.52 & 0.09 & 3.33 & $7.1 \pm 1.6$ \\
$19265013+0149070$ & 4770 & 42 & 2.68 & 0.09 & -0.50 & 0.25 & - & - & 3.68 & 0.14 & 3.32 & $13.2 \pm 1.7$ \\
$19265193+0044004$ & 4880 & 58 & 2.54 & 0.11 & -0.33 & 0.13 & 1.50 & 0.22 & 2.94 & 0.06 & 2.80 & $2.1 \pm 2.7$ \\
$19270600+0134446$ & 4584 & 36 & 2.38 & 0.09 & +0.19 & 0.17 & - & - & 3.67 & 0.13 & 3.53 & $\leq 7.0$ \\
$19270815+0017461$ & 4514 & 41 & 2.28 & 0.09 & -0.29 & 0.26 & - & - & 2.33 & 0.08 & 2.37 & $7.8 \pm 1.6$ \\
$19273856+0024149$ & 4446 & 38 & 2.39 & 0.09 & -0.16 & 0.21 & - & - & 2.33 & 0.16 & 2.37 & $12.0 \pm 0.5$ \\
$19274706+0023447$ & 4608 & 66 & 3.21 & 0.13 & +0.13 & 0.24 & - & - & 2.78 & 0.14 & 2.70 & $18.0 \pm 0.5$ \\
$19280508+0100139$ & 4623 & 38 & 2.49 & 0.09 & +0.11 & 0.18 & - & - & 3.66 & 0.10 & 3.49 & $8.2 \pm 1.5$ \\
$19283226+0033072$ & 4600 & 38 & 2.64 & 0.09 & +0.29 & 0.21 & - & - & 3.60 & - & 3.46 & $\leq 7.0$ \\
\hline
\end{tabular}

Notes. In the case of stars with GIRAFFE spectra, the errors in the parameters and abundances are the standard deviation of values obtained using multiple analysis pipelines. These are thus estimates of the internal error alone. The missing error of some Li abundances means that the values were determined by one single pipeline. Assuming a typical error of $\sim 0.10-0.15 \mathrm{dex}$ (as for the other values) would be adequate in these cases. In the case of stars with UVES spectra, errors are obtained through modeling of how well the multiple pipelines reproduce the reference parameters of calibrating objects (e.g., Gaia benchmark stars). The process of error estimation in the analysis of UVES spectra will be described in Casey et al. (in prep). For stars observed with GIRAFFE, values of $\mathrm{v} \sin i$ are reported only if above $\sim 7.0 \mathrm{~km} \mathrm{~s}^{-1}$. For these measurements, the HR15N setup (centred at $665 \mathrm{~nm}$ ) was used. 


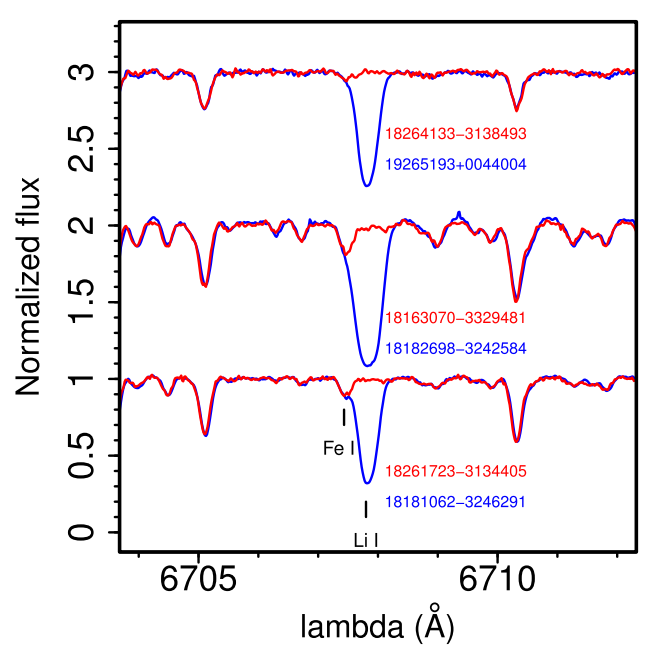

Fig. 1. Spectra of three Li-rich giants at the wavelength of the Li $6708 \AA$ line (in blue). In each case, the spectrum of a comparison giant with similar atmospheric parameters is also shown (in red; similar means within $\pm 50 \mathrm{~K}$ in $T_{\text {eff }}$ and $\pm 0.10 \mathrm{dex}$ in $\log g$ and $\left.[\mathrm{Fe} / \mathrm{H}]\right)$. The flux has been normalised and arbitrarily shifted to facilitate the visualisation.

Originally, 21 candidate Li-rich giants were identified. Upon inspection of the spectra, however, one giant was found to lack an enhanced Li line (CNAME 08113284-4721004) and was excluded from further discussion. Its enhanced $\mathrm{Li}$ abundance is likely an artefact introduced by some failure of the analysis pipelines. We have checked the literature, and to the best of our knowledge, all 20 giants are reported to be Li-rich here for the first time.

Corrections for non-LTE effects were applied using the grid of Lind et al. (2009). The corrections depend on the atmospheric parameters and on the LTE Li abundance. They are usually positive if the $\mathrm{Li}$ enrichment is not too high (around $2.0 \mathrm{dex}$ ), but may become negative for the giants with $\mathrm{Li}$ abundances above $\sim 2.6$ dex. For the stars observed with GIRAFFE, a spectroscopic determination of the microturbulence velocity was not possible. For the purposes of the non-LTE correction, a value of $\xi=1.5 \mathrm{~km} \mathrm{~s}^{-1}$ was adopted as is reasonable for red giants. In any case, the non-LTE correction is not very sensitive to $\xi$. The LTE and non-LTE Li abundances are given in Table 2.

\section{Properties of the new Li-rich giants}

Within the Gaia-ESO data analysis cycle, a detailed system of flags has been defined with which any peculiarity identified in the spectra and/or issues found during the analysis can be reported. It is anticipated that a number of these flags will be included in later public data releases (flags to be described in Van Eck et al., in prep). For the moment, we can report that no important problem with the spectra of these Li-rich giants has been flagged (e.g. no emission lines, and no evidence of multiplicity in the spectra). For the method developed to identify spectroscopic binaries, we refer to the discussion presented in Merle et al. (2017).

The subsections below report our investigation of the properties of the new Li-rich giants (e.g. core of strong lines for signs of magnetic activity, infrared (IR) magnitudes, rotational velocities, chemical abundances, and Galactic velocities). We attempted to identify characteristics in common among these stars. A summary of these findings is given in Table 3.

\subsection{Position in the $T_{\mathrm{eff}}-\log g$ diagram}

Figure 2 displays the newly discovered Li-rich giants in $T_{\text {eff }}{ }^{-}$ $\log g$ diagrams, where the giants are divided according to metallicity. A selection of evolutionary tracks for masses between 0.8 and $3.0 M_{\odot}$ is shown for comparison. The regions of the RGB luminosity bump, the clump of low-mass stars, and of the beginning of the early-AGB are indicated. Low-mass stars here are those stars that go through the He-core flash at the end of the RGB. The beginning of the early-AGB is defined here as the first point where the luminosity produced by the He-burning shell becomes higher than the luminosity produced by the H-burning shell, at the evolutionary stages after the abundance of central He has reached zero.

For masses below $1.4 M_{\odot}$, we use the new PARSEC evolutionary tracks of Fu et al. (2018) which were computed with a new envelope overshooting calibration. As a result, the RGB bump, for these tracks, is shifted by between +0.15 or +0.20 dex in $\log g$ in comparison with the older PARSEC tracks. For masses above $1.4 M_{\odot}$, we plot the older PARSEC tracks (as the position of the RGB bump was not changed in the new tracks).

Charbonnel \& Balachandran (2000) suggested that Li-rich giants were preferentially located in two regions of the HR diagram. Low-mass Li-rich giants would be located at the RGB bump, and intermediate-mass Li-rich giants would be located at the early AGB. Charbonnel \& Balachandran (2000) connected the $\mathrm{Li}$ enrichment with an extra-mixing process that activates at these evolutionary stages.

More recently, some Li-rich giants were found to be instead core He-burning giants (Kumar et al. 2011; Monaco et al. 2014; Silva Aguirre et al. 2014; Bharat Kumar et al. 2018). There are, however, works that report Li-rich giants throughout the RGB (see, e.g. Alcalá et al. 2011; Monaco et al. 2011; Martell \& Shetrone 2013) and others that find Li-rich objects also among less evolved stars (Koch et al. 2011; Gruyters et al. 2016; Li et al. 2018).

Figure 2 shows at least one giant (18265248+0627259) close to the position of the core He-burning stage at the intermediatemass regime (in the bottom left panel). This star is potentially very interesting. To explain a concentration of $\mathrm{Li}$-rich giants around the clump, Kumar et al. (2011) suggested an episode of $\mathrm{Li}$ production related to the He-core flash. However, such intermediate-mass giants do not go through the He-core flash and would thus require a different scenario for the Li enrichment. Nevertheless, this star is a fast rotator (see Sect. 3.3 below) and has quite uncertain parameters. Thus, it might also be a low-mass clump giant.

Within the errors, the position of most giants in Fig. 2 is consistent with the RGB luminosity bump or the clump of low-mass stars. This is true in all metallicity intervals. There are maybe five giants (one in the top middle panel, two in the bottom left panel, and two in the bottom middle panel) that fall either above or below the bump. For at least four of them, we consider the parameters to be uncertain. These four stars out of the five have spectra with signal-to-noise (S/N) below 30 (see Table 1). They might have been excluded from the discussion, but we chose to report them here, nonetheless, because they have enhanced $\mathrm{Li}$ lines that make them genuine Li-rich giants.

Given the error bars, a few of the giants might instead be in the early-AGB region, if they are of intermediate mass. The error bars do not allow us to clearly classify them as low- or intermediate-mass giants. Despite the difficulty in assigning a specific evolutionary stage, it seems clear from Fig. 2 that the 
Table 3. Summary of the noteworthy characteristics of the new Li-rich giants as discussed in Sects. 3 and 4.

\begin{tabular}{|c|c|}
\hline CNAME & Characteristics \\
\hline \multirow{2}{*}{\multicolumn{2}{|c|}{ Above the bump in both the $T_{\text {eff }}-\log g$ diagram (Fig. 2 ) and the HR-diagram (Fig. 7 ); bottom left panels. }} \\
\hline & \\
\hline \multicolumn{2}{|l|}{$17531013-2932063$} \\
\hline \multicolumn{2}{|l|}{$18181062-3246291$} \\
\hline $18182698-3242584$ & $\alpha$-enhanced with super-solar metallicity. \\
\hline $18265248+0627259$ & $\begin{array}{l}\text { Possible core-He burning intermediate-mass giant? (bottom left panel of Fig. 2); candidate for } \\
\text { planet engulfment (fast rotation); closer to the clump in the HR-diagram (Fig. 7). }\end{array}$ \\
\hline \multicolumn{2}{|l|}{$19223053+0138518$} \\
\hline \multicolumn{2}{|l|}{$19251759+0053140$} \\
\hline \multirow{2}{*}{\multicolumn{2}{|c|}{$\begin{array}{l}19261134+0051569 \\
19263808+0054441\end{array}$}} \\
\hline & \\
\hline \multicolumn{2}{|l|}{$19264134+0137595$} \\
\hline $19264917-0027469$ & $\begin{array}{l}\left.\text { Above the bump ( } T_{\mathrm{eff}}-\log g \text { diagram, bottom-middle panel of Fig. } 2\right) ; S / N<30 ;[\alpha / \mathrm{Fe}]>+0.15 \text {; } \\
\text { luminosity not computed. }\end{array}$ \\
\hline $19265013+0149070$ & {$[\alpha / \mathrm{Fe}]>+0.15 ; \mathrm{Ba}$ enhanced (mass transfer from an AGB?). } \\
\hline $19265193+0044004$ & Core-He burning giant from seismic data. \\
\hline $19270600+0134446$ & Infrared excess at $22 \mu \mathrm{m}$ \\
\hline $19270815+0017461$ & $\begin{array}{l}\left.\text { Above the bump ( } T_{\text {eff }}-\log g \text { diagram, bottom left panel of Fig. } 2\right) ; S / N<30 ;[\alpha / \mathrm{Fe}]>+0.15 \text {; } \\
\text { luminosity not computed. }\end{array}$ \\
\hline $19273856+0024149$ & $\begin{array}{l}\left.\text { Above the bump ( } T_{\text {eff }}-\log g \text { diagram, bottom left panel of Fig. } 2\right) ; S / N<30 ;[\alpha / \mathrm{Fe}]>+0.15 \text {; } \\
\text { luminosity not computed. }\end{array}$ \\
\hline $19274706+0023447$ & $\begin{array}{l}\left.\text { Below the bump ( } T_{\text {eff }}-\log g \text { diagram, top middle panel of Fig. } 2\right) ; S / N<30 \text {; candidate for planet } \\
\text { engulfment (fast rotation); at the clump in the HR-diagram (top middle panel of Fig. } 7 \text { ). }\end{array}$ \\
\hline \multicolumn{2}{|l|}{$19280508+0100139$} \\
\hline $19283226+0033072$ & \\
\hline
\end{tabular}

Li-rich giants are found in a narrow and specific region of the diagram.

In general, the position of giants in such spectroscopic diagrams can be quite uncertain. A plot such as we show in Fig. 2 is not sufficient to tell the evolutionary stages apart. Photometric diagrams tend to be more precise if the distance to the star and the reddening are well known. We resume this discussion using Gaia DR2 parallaxes in Sect. 4.3. Otherwise, asteroseismology is the only way to properly separate the evolutionary stages (Bedding et al. 2011; Mosser et al. 2011; Elsworth et al. 2017). The stellar properties based on CoRoT data, which are available for some of our giants, are discussed in Sects. 4.1 and 4.2.

\subsection{Stellar masses}

Mints \& Hekker (2017) recently presented a tool for estimating masses, ages, and distances of stars using a Bayesian approach (UniDAM, the unified tool to estimate distances, ages, and masses). The tool makes use of PARSEC isochrones (Bressan et al. 2012) and needs as input the atmospheric parameters $\left(T_{\text {eff }}, \log g\right.$, and $\left.[\mathrm{Fe} / \mathrm{H}]\right)$ and the IR photometry from 2MASS (Two Micron All-Sky Survey, Skrutskie et al. 2006) and WISE (Wide-field Infrared Survey Explorer, Wright et al. 2010; Cutri \& et al. 2012, 2013).

Using UniDAM, Mints \& Hekker (2017) estimated the mass of one star of our sample, CNAME 19223053+0138518, using Gaia-ESO DR2 atmospheric parameters. They derived mean masses of 1.31 or $1.50( \pm 0.21) M_{\odot}$, depending on whether the star was assumed to be before or during He-core burning.

We have employed UniDAM to obtain an indicative value of the stellar masses in our sample. We used the atmospheric parameters from Table 2 combined with $J, H$, and $K s$ magnitudes from 2MASS, and $W 1$ and $W 2$ magnitudes from WISE. All magnitudes were retrieved from the Vizier database (Ochsenbein et al. 2000). The results are given in Table 4 (and also include age and distance estimates). For a few stars the solutions did not converge or were flagged as being of low quality, and are thus not given. Estimates are not provided for star CNAME 17531013-2932063 as it has saturated 2MASS magnitudes. Some discrepancy between the masses estimated using UniDAM and what would be estimated by eye from Fig. 2 can be noted. This is likely caused by the additional use of IR photometry in the calculations. Within the errors, however, the estimates of stellar masses would be in agreement. Moreover, we tested UniDAM in a series of giants in open clusters observed by Gaia-ESO with ages between 0.3 and 4.5 Gyr. The cluster ages and stellar masses obtained by UniDAM were in agreement, within the errors, with the known properties of the clusters.

The UniDAM code typically outputs two (sometimes three) solutions, assuming that the giants are in different evolutionary stage. Most Li-rich giants seem to be low-mass stars between 1.1 and $1.4 M_{\odot}$, either before or during core-He burning, and thus either at the RGB luminosity bump or at the clump.

We remark that this method does not use any seismic information, but only spectroscopic and photometric observables and theoretical isochrones. It is well known that such mass estimates for red giants are affected by large uncertainties, given the accumulation of model tracks with different masses in a small region of the HR diagram and uncertainties in the chemical composition. In Sect. 4.1 below, we also discuss estimates of stellar masses based on seismic properties. Although these values also 

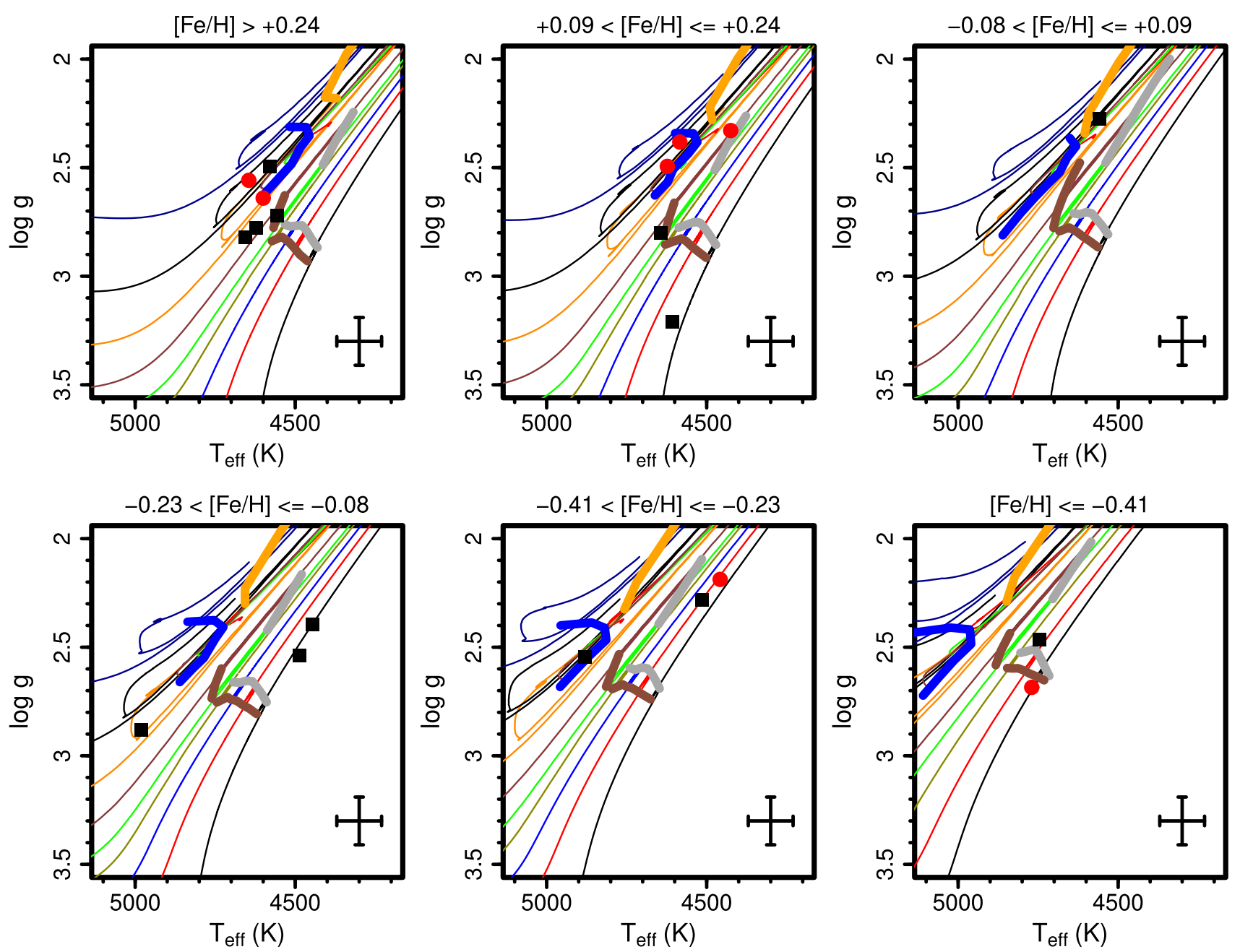

Fig. 2. Newly discovered Li-rich giants in the $T_{\text {eff }}-\log g$ diagram divided according to metallicity into different panels. PARSEC evolutionary tracks (Bressan et al. 2012; Fu et al. 2018) of masses 0.8, 1.0, 1.2, 1.4, 1.5, 1.7, 2.0, 2.4, and 3.0 $M_{\odot}$ are shown. From the top left to the bottom right panels, the isochrones have $[\mathrm{Fe} / \mathrm{H}]=+0.30,+0.18,0.00,-0.15,-0.30$, and -0.52 dex. The range of $[\mathrm{Fe} / \mathrm{H}]$ of the stars is given at the top of each panel. The beginning and the end of the RGB luminosity bump are marked as thick grey and brown lines, respectively. The position of the clump of low-mass giants is shown as a thick blue line. The beginning of the early-AGB of intermediate-mass stars is highlighted as the thick orange line. Super Li-rich giants with $A(\mathrm{Li})>3.3 \mathrm{dex}$ (in non-LTE) are shown as red circles, giants with $\mathrm{Li}$ abundance below this are shown as black squares. For the intermediate-mass stars, tracks are plotted only until the point where central He reaches approximately zero (end of core-He burning). Typical error bars $\left( \pm 70 \mathrm{~K}\right.$ in $T_{\text {eff }}$ and \pm 0.10 dex in $\log g$ ) are shown in the bottom right corner of the panels.

suffer from large uncertainties, the estimates seem to be consistent within the errors.

\subsection{Activity, infrared excess, and rotation}

Lithium-rich giants are sometimes found as by-products in searches for young stars that use X-ray detection, IR excess, or chromospheric activity as selection criteria (e.g. Gregorio-Hetem et al. 1993; Castilho et al. 1998; Frasca et al. 2018). Several works have reported detection of magnetic activity in some Li-rich giants (see, e.g. Kővári et al. 2013, 2017; Kriskovics et al. 2014). To search for evidence of activity, we investigated the stellar spectra for signs of emission in the core of strong lines ( $\mathrm{H} \alpha$ and the near-IR Ca II lines). No clear signs of emission were found in any of the giants.

The IR excess that is sometimes reported in Li-rich giants has been suggested to be connected to an episode of enhanced mass loss (de la Reza et al. 1996, 1997, 2015). However, investigation of large samples of Li-rich and Li-normal giants has shown that IR excess seems to be rare (Jasniewicz et al. 1999;
Bharat Kumar et al. 2015; Rebull et al. 2015). This indicates that either the possible mass-loss event is very short lived or that there is no connection between mass loss and $\mathrm{Li}$ enrichment.

We have investigated the IR behaviour of our Li-rich giants using 2MASS and WISE photometry. For comparison, a flux model of each star was computed using Kurucz codes (Kurucz 1993). The modelled $\log$ (wavelength $\times$ flux) was normalised to the $J$ band and compared to the remaining magnitudes. The agreement between models and observations is very good for all bands from $H$ to W3. For most stars, the WISE $W 4$ band (at $22 \mu \mathrm{m}$ ) is only an upper limit. Only two stars have W4 detections. For star CNAME 19223053+0138518 (CoRoT 100440565), the agreement with the model is good. For star CNAME 19270600+0134446 (CoRoT 101205220), an excess emission is indicated at $22 \mu \mathrm{m}$ (Fig. 3). Given the lack of $W 4$ magnitudes for most stars in our sample, we do not have a clear picture of how common the IR excess is in these new Li-rich giants.

Projected rotational velocities are listed in Table 2. Many of the giants have only upper limits determined from their 
Table 4. Masses, ages, and distances estimated using UniDAM.

\begin{tabular}{lcccc}
\hline \hline CNAME & $\begin{array}{c}\text { Mass } \\
\left(M_{\odot}\right)\end{array}$ & $\begin{array}{c}\log (\text { Age }) \\
(\mathrm{Gyr})\end{array}$ & $\begin{array}{c}\text { Distance } \\
(\mathrm{kpc})\end{array}$ & Stage \\
\hline $08405643-5308309$ & $1.2 \pm 0.3$ & $9.7 \pm 0.3$ & $2.5-2.7 \pm 0.5$ & I or II \\
$17522490-2927512$ & - & - & - & - \\
$17531013-2932063$ & - & - & - & - \\
$18181062-3246291$ & $1.2-2.1 \pm 0.5$ & $9.1-9.8 \pm 0.4$ & $1.7-2.6 \pm 0.5$ & I, II or III \\
$18182698-3242584$ & - & - & - & - \\
$18265248+0627259$ & $1.2-1.9 \pm 0.5$ & $9.3-9.8 \pm 0.3$ & $2.5-3.8 \pm 0.7$ & I or II \\
$19223053+0138518$ & $1.4 \pm 0.4$ & $9.6 \pm 0.3$ & $1.5-1.6 \pm 0.2$ & I or II \\
$19251759+0053140$ & $1.4-1.9 \pm 0.2$ & $9.2-9.6 \pm 0.2$ & $1.9-2.6 \pm 0.3$ & I or II \\
$19261134+0051569$ & $1.2 \pm 0.3$ & $9.7 \pm 0.3$ & $4.0 \pm 0.6$ & I or II \\
$19263808+0054441$ & $1.4-1.9 \pm 0.2$ & $9.2-9.6 \pm 0.2$ & $1.0-1.4 \pm 0.2$ & I or II \\
$19264134+0137595$ & $1.6-2.6 \pm 0.3$ & $8.9-9.5 \pm 0.2$ & $2.9-3.8 \pm 0.5$ & II \\
$19264917-0027469$ & $1.3-1.9 \pm 0.3$ & $9.2-9.7 \pm 0.3$ & $8.5-11.6 \pm 0.1$ & I or III \\
$19265013+0149070$ & $1.2-1.7 \pm 0.3$ & $9.4-9.7 \pm 0.3$ & $4.2-5.4 \pm 0.7$ & I or II \\
$19265193+0044004$ & $1.3-1.4 \pm 0.4$ & $9.5-9.6 \pm 0.3$ & $1.8-1.9 \pm 0.3$ & I or II \\
$19270600+0134446$ & $1.1-1.6 \pm 0.4$ & $9.5-9.8 \pm 0.3$ & $3.5-3.9 \pm 0.8$ & I or II \\
$19270815+0017461$ & $1.2-1.3 \pm 0.4$ & $9.7-9.8 \pm 0.3$ & $5.1-5.5 \pm 1.0$ & I or II \\
$19273856+0024149$ & $1.1-1.2 \pm 0.2$ & $9.8-9.9 \pm 0.2$ & $4.3-4.5 \pm 0.6$ & I or II \\
$19274706+0023447$ & - & - & - & - \\
$19280508+0100139$ & $1.3-1.4 \pm 0.4$ & $9.6-9.7 \pm 0.3$ & $4.6-4.9 \pm 0.7$ & I or II \\
$19283226+0033072$ & $1.3 \pm 0.2$ & $9.7 \pm 0.2$ & $2.7 \pm 0.4$ & I \\
\hline
\end{tabular}

Notes. We adopt the mean values of mass, age, and distance and the standard deviation, but note that other estimates are also provided by UniDAM (e.g. mode and median). Usually, UniDAM returns two (or three) estimates per star, assuming different evolutionary stages - I: pre He-core burning; II: during He-core burning, or III: post He-core burning. The stages are given in the last column. When the estimates per stage are different, we list all values, otherwise only one value is given. Solutions of low quality (low probability) are discarded.

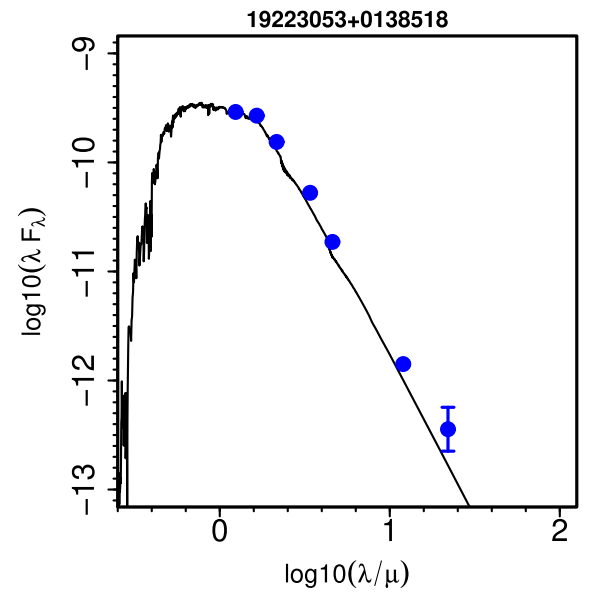

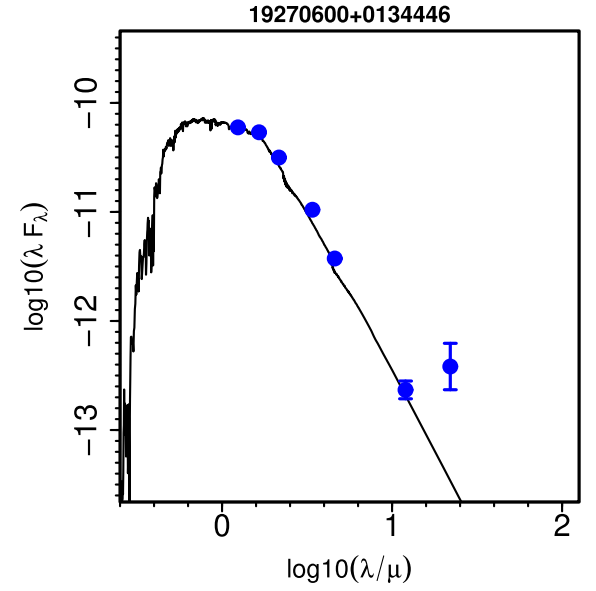

Fig. 3. Comparison between synthetic flux model and magnitudes (2MASS and WISE) for two stars, 19223053+0138518 (left) and 19270600+0134446 (right). Star $19270600+0134446$ seems to have excess emission at the $W 4$ band $(22 \mu \mathrm{m})$.
GIRAFFE spectra and are thus likely slow rotators, including $19270600+0134446$, the only giant with IR excess. Nine giants, however, have $\mathrm{v} \sin i>7-8 \mathrm{~km} \mathrm{~s}^{-1}$. This seems to agree with the results of Drake et al. (2002), who found that Li-rich giants are more common among fast-rotating giants (defined by them as giants with $\mathrm{v} \sin i>8 \mathrm{~km} \mathrm{~s}^{-1}$ ).

Fast rotation is one of the expected outcomes of planet engulfment (e.g. Carlberg et al. 2009). The high $v \sin i$ of some of the Li-rich giants could thus be interpreted as a sign of engulfment. Privitera et al. (2016a,b) computed models that take into account the interaction between the planetary orbit and rotation in stars during engulfment episodes. In their rotating stellar models, equatorial velocities (not projected) of the order of $5-10 \mathrm{~km} \mathrm{~s}^{-1}$ are possible for giants of $1.5 M_{\odot}$ and $\log g \sim 2.5$ dex, even without engulfment, just from the normal spin-down evolution of the star. Only higher v $\sin i$ values would need to be explained with some sort of acceleration of the stellar surface.

Two stars in our sample could be examples of such cases. One is star 19274706+0023447 (CoRoT 101314825), the giant at the lower RGB (top middle panel of Fig. 2), which has $18 \mathrm{~km} \mathrm{~s}^{-1}$. The other is star $18265248+0627259$, the fastest rotator in this sample with $37 \mathrm{~km} \mathrm{~s}^{-1}$ (the star apparently at the core-He burning phase, bottom left panel of Fig. 2). In light of the work of Privitera et al. (2016a,b), both stars might have suffered surface acceleration because of the engulfment of planets. We note that this last star was observed in the field of the open cluster NGC 6633 (with ID NGC 6633 JEF 49), but it is not a member based on its radial velocity (RV) and photometry (Jeffries 1997, who also measured $v \sin i=39 \mathrm{~km} \mathrm{~s}^{-1}$ ). 
Table 5. Selected chemical abundances of the Li-rich giants.

\begin{tabular}{lcccccc}
\hline \hline CNAME & {$[\alpha / \mathrm{Fe}]$} & {$[\mathrm{Mg} / \mathrm{Fe}]$} & {$[\mathrm{Al} / \mathrm{Fe}]$} & {$[\mathrm{Na} / \mathrm{Fe}]$} & {$[\mathrm{Ba} / \mathrm{Fe}]$} & {$[\mathrm{Eu} / \mathrm{Fe}]$} \\
\hline $08405643-5308309$ & - & - & - & - & - & - \\
$17522490-2927512$ & $-0.17 \pm 0.17$ & - & $+0.21 \pm 0.07$ & - & - & - \\
$17531013-2932063$ & $+0.18 \pm 0.13$ & - & $+0.13 \pm 0.06$ & - & - & - \\
$18181062-3246291$ & $-0.01 \pm 0.08$ & $-0.02 \pm 0.05$ & $-0.04 \pm 0.03$ & $-0.07 \pm 0.09$ & $+0.02 \pm 0.13$ & $-0.14 \pm 0.06$ \\
$18182698-3242584$ & $+0.22 \pm 0.16$ & $+0.22 \pm 0.12$ & $+0.32 \pm 0.08$ & $+0.37 \pm 0.29$ & $+0.01 \pm 0.01$ & $-0.04 \pm 0.11$ \\
$18265248+0627259$ & - & - & - & - & - & - \\
$19223053+0138518$ & $+0.05 \pm 0.23$ & $+0.00 \pm 0.04$ & $+0.26 \pm 0.05$ & - & - & - \\
$19251759+0053140$ & $-0.12 \pm 0.20$ & $-0.09 \pm 0.03$ & $+0.07 \pm 0.05$ & - & - & - \\
$19261134+0051569$ & $+0.39 \pm 0.22$ & $+0.37 \pm 0.04$ & $+0.34 \pm 0.05$ & - & $+0.23 \pm 0.21$ & - \\
$19263808+0054441$ & $-0.09 \pm 0.12$ & $+0.01 \pm 0.02$ & $+0.11 \pm 0.03$ & - & - & - \\
$19264134+0137595$ & $+0.06 \pm 0.20$ & $+0.07 \pm 0.03$ & $+0.18 \pm 0.04$ & - & - & - \\
$19264917-0027469$ & $+0.40 \pm 0.26$ & $+0.34 \pm 0.04$ & $+0.52 \pm 0.10$ & - & $-0.20 \pm 0.24$ & - \\
$19265013+0149070$ & $+0.36 \pm 0.25$ & $+0.30 \pm 0.04$ & $+0.34 \pm 0.05$ & - & $+0.77 \pm 0.24$ & - \\
$19265193+0044004$ & $+0.05 \pm 0.13$ & $+0.04 \pm 0.08$ & $+0.05 \pm 0.05$ & $0.00 \pm 0.13$ & $-0.10 \pm 0.03$ & $-0.06 \pm 0.02$ \\
$19270600+0134446$ & $-0.03 \pm 0.17$ & $-0.05 \pm 0.03$ & $+0.22 \pm 0.05$ & - & - & - \\
$19270815+0017461$ & $+0.16 \pm 0.26$ & $+0.16 \pm 0.04$ & $+0.29 \pm 0.05$ & - & $+0.13 \pm 0.22$ & - \\
$19273856+0024149$ & $+0.19 \pm 0.22$ & $+0.12 \pm 0.04$ & $+0.17 \pm 0.05$ & - & - & $+0.11 \pm 0.06$ \\
$19274706+0023447$ & $+0.07 \pm 0.24$ & $-0.03 \pm 0.03$ & $+0.12 \pm 0.05$ & - & - & $+0.27 \pm 0.06$ \\
$19280508+0100139$ & $+0.05 \pm 0.18$ & $-0.01 \pm 0.03$ & $+0.12 \pm 0.05$ & - & - & $+0.01 \pm 0.06$ \\
$19283226+0033072$ & $-0.06 \pm 0.22$ & $-0.05 \pm 0.03$ & $+0.13 \pm 0.05$ & - & - & - \\
\hline
\end{tabular}

Notes. Solar abundances of $\mathrm{Mg}, \mathrm{Al}, \mathrm{Na}, \mathrm{Ba}$, and Eu were adopted from Grevesse et al. (2007). The abundance errors have the same meaning as discussed in the note of Table 2 .

Table 6. Abundances of $\mathrm{C}, \mathrm{N}$, and $\mathrm{O}$ in the three Li-rich giants observed with UVES.

\begin{tabular}{lccc}
\hline \hline CNAME & {$[\mathrm{C} / \mathrm{Fe}]$} & {$[\mathrm{N} / \mathrm{Fe}]$} & {$[\mathrm{O} / \mathrm{Fe}]$} \\
\hline $18181062-3246291$ & $-0.17 \pm 0.03$ & $+0.01 \pm 0.06$ & $-0.02 \pm 0.05$ \\
$18182698-3242584$ & $-0.03 \pm 0.04$ & $+0.46 \pm 0.06$ & $+0.01 \pm 0.05$ \\
$19265193+0044004$ & $-0.21 \pm 0.06$ & $+0.23 \pm 0.05$ & $-0.10 \pm 0.03$ \\
\hline
\end{tabular}

Notes. Solar abundances of $\mathrm{C}, \mathrm{N}$, and $\mathrm{O}$ were adopted from Grevesse \& Sauval (1998). We gave preference to these older values for CNO because Grevesse et al. (2007) list abundances derived using 3D models, which considerably decrease the value of the reference solar abundances. Our analysis, however, is based on 1D models, and thus Grevesse \& Sauval (1998) offer more consistent reference values. All abundances are given in LTE. The abundance errors have the same meaning as discussed in the note of Table 2.

\subsection{Chemical abundances}

We have checked the chemical abundances of other elements available in iDR5 for possible anomalies. Abundances of a few selected elements in the Li-rich giants are given in Table 5. The abundance information for the stars observed with GIRAFFE is limited because of the restricted wavelength range of the spectra (see Mikolaitis et al. 2014). Abundances of C, N, and O are only available for giants observed with UVES and are given in Table 6.

Surface abundances of $\mathrm{C}$ and $\mathrm{N}$ are expected to be altered in giants that have gone through the first dredge-up; $[\mathrm{C} / \mathrm{Fe}]$ $\sim-0.20$ dex and $[\mathrm{N} / \mathrm{Fe}] \sim+0.40$ dex (see, e.g. Tautvaišienè et al. 2015, 2016; Drazdauskas et al. 2016a,b; Böcek Topcu et al. 2015, 2016; Szigeti et al. 2018, for some recent references). The $\mathrm{C}$ and $\mathrm{N}$ abundances seem consistent with the stars having experienced first dredge-up, although $\mathrm{N}$ in the star CNAME 18181062-3246291 seems to have been only mildly affected.
Abundances of other elements $(\mathrm{Na}, \mathrm{Al}, \alpha$, iron peak, and neutron capture) were also investigated. Of the heavy neutroncapture elements, Table 5 only lists $[\mathrm{Ba} / \mathrm{Fe}]$ and $[\mathrm{Eu} / \mathrm{Fe}]$ values. The only peculiarity that was identified lies in the $[\mathrm{Ba} / \mathrm{Fe}]$ ratio of star CNAME 19265013+0149070 (CoRoT 101162874), which is clearly enhanced. We have double-checked the Ba abundance of this star using spectrum synthesis and confirm the reported enhancement. We remark that this star is rotating moderately fast $\left(v \sin i=13.2 \mathrm{~km} \mathrm{~s}^{-1}\right.$, see Table 2$)$. The Ba enhancement might suggest that this could be a barium giant (see, e.g. de Castro et al. 2016; Escorza et al. 2017). Barium giants are members of binary systems, with companions that are currently white dwarfs. The more massive companion evolved faster, went through the AGB phase and enriched itself with neutron capture elements, before transferring mass to the current Ba giant. The $\mathrm{Li}$ enhancement might also originate from the AGB companion. However, there seems to be no clear evidence of other Li-rich Ba giants (Lambert et al. 1993).

\subsection{Stellar population analysis}

The second data release (DR2) of Gaia has provided astrometric information for more than $1.3 \times 10^{9}$ objects with unprecedented quality (Gaia Collaboration 2016, 2018; Lindegren et al. 2018). We have cross-matched our list of Li-rich giants with Gaia DR2 and obtained parallaxes and proper motions for all the stars (Table A.1).

The relative uncertainty of the parallaxes has median of about $10 \%$ and is lower than $25 \%$ for most stars. For this preliminary kinematic analysis, we assumed that the stellar distance is the inverse of the parallax. This assumption should provide accurate results for most of our stars, but not for all of them. We refer to Luri et al. (2018) for a discussion of the uncertainties, correlations, and limitations of the parallaxes. We considered only giants whose relative uncertainty of the parallaxes is lower than $15 \%$. 

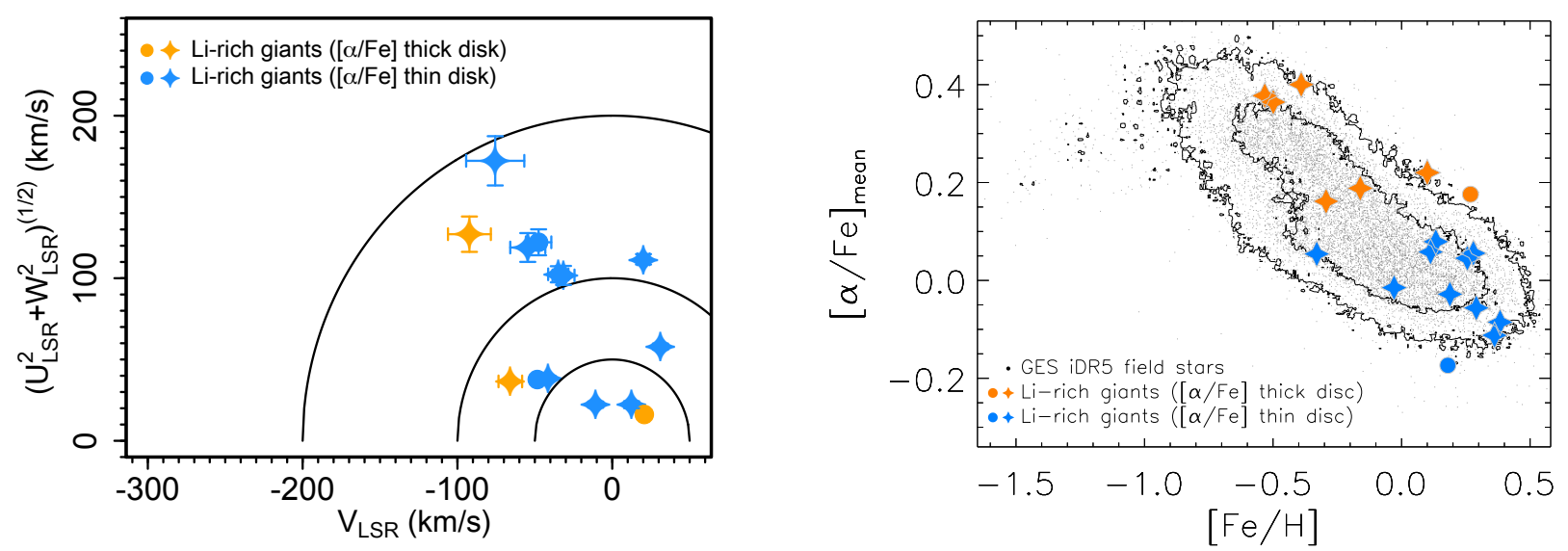

Fig. 4. Left: Toomre diagram of the new Li-rich giants with good Gaia DR2 parallaxes (i.e. only the 14 giants with a relative error of the parallax lower than 15\%). Error bars are shown, but in many cases they are smaller than the point size. Right: chemical plot of the $[\alpha / \mathrm{Fe}]$ ratio as a function of $[\mathrm{Fe} / \mathrm{H}]$. In all plots, the field Li-rich giants are displayed as stars and giants in fields of open clusters as solid circles. The colour is orange or blue for stars that are tentatively associated with the thick or thin disc, respectively. Other Gaia-ESO iDR5 field stars are shown as dots in the right panel.

The calculation of the heliocentric Galactic space-velocity components $(U, V$, and $W)$ and respective uncertainties is based on the equations presented in Johnson \& Soderblom (1987). The components are in the right-hand system, meaning that $U$ is positive towards the Galactic centre, $V$ is positive towards the Galactic rotation, and $W$ is positive towards the Galactic north pole. For this calculation, we assumed that the local standard of rest rotates with $V_{\mathrm{LSR}}=220 \mathrm{~km} \mathrm{~s}^{-1}$ and adopted the 3D solar motion of $(U, V, W)_{\odot}=(+10.0,+5.2,+7.2)$ from Dehnen \& Binney (1998).

The results are displayed in the left panel of Fig. 4 and are listed in Table 7. The velocities of the Li-rich giants do not deviate significantly from the behaviour expected of stars in the Galactic disc. In the right panel, we include the plot of the $[\alpha / \mathrm{Fe}]$ ratio as a function of $[\mathrm{Fe} / \mathrm{H}]$. The value of $[\alpha / \mathrm{Fe}]$ is an average of abundances of $[\mathrm{Mg} \mathrm{i} / \mathrm{Fe}],[\mathrm{Si} \mathrm{i} / \mathrm{Fe}],[\mathrm{Ca} \mathrm{i} / \mathrm{Fe}],[\mathrm{Ti} \mathrm{i} / \mathrm{Fe}]$, and $[\mathrm{Ti}$ II/Fe]. The Li-rich giants are compared to the field stars included in the iDR5 catalogue. In this last panel, the dots are results of Monte Carlo Markov chain (MCMC) simulations that take into account the uncertainties in the measured abundances. The contours in mark regions containing $95 \%$ and $68 \%$ of the data points. Using their $[\alpha / \mathrm{Fe}]$ ratios, we tentatively classify 11 giants as members of the thin disc and seven as members of the thick disc. No $\alpha$-element abundances are available for the remaining two giants. We note, however, that the tentative thin- and thick-disc giants do not separate well in the kinematic plot.

One of the tentative thick-disc stars, 18182698-3242584, was observed in a field towards the bulge, and thus it might instead be a member of this stellar population (but see Appendix D below), in particular, given the known chemical similarity between bulge and thick disc (Alves-Brito et al. 2010). Alternatively, it might also be a member of the $\alpha$-enhanced super-solar metallicity population identified by Adibekyan et al. (2011). A detailed discussion of the kinematic properties of the sample is beyond the scope of this paper, but we can conclude that they mostly seem to be disc giants.

\section{Discussion}

In this section, we attempt to address the evolutionary stage of the stars in more detail. To do this, we make use of the CoRoT
Table 7. Galactic space velocities for the new sample of Li-rich giants reported in this work.

\begin{tabular}{crrr}
\hline \hline CNAME & \multicolumn{1}{c}{$U$} & \multicolumn{1}{c}{$V$} & \multicolumn{1}{c}{$W$} \\
$\mathrm{~km} \mathrm{~s}^{-1}$ & \multicolumn{1}{c}{$\mathrm{km} \mathrm{s}^{-1}$} & \multicolumn{1}{c}{$\mathrm{km} \mathrm{s}^{-1}$} \\
\hline $08405643-5308309$ & $-36.2 \pm 2.0$ & $-48.4 \pm 0.3$ & $-10.5 \pm 0.6$ \\
$17522490-2927512$ & - & - & - \\
$17531013-2932063$ & $-16.1 \pm 0.2$ & $20.7 \pm 2.2$ & $1.8 \pm 1.2$ \\
$18181062-3246291$ & $43.5 \pm 0.5$ & $31.1 \pm 1.8$ & $-38.3 \pm 2.8$ \\
$18182698-3242584$ & $36.1 \pm 0.4$ & $-65.8 \pm 7.6$ & $-5.6 \pm 1.2$ \\
$18265248+0627259$ & $102.5 \pm 7.6$ & $-47.6 \pm 8.2$ & $-66.3 \pm 9.0$ \\
$19223053+0138518$ & $-11.5 \pm 0.4$ & $-10.7 \pm 0.5$ & $-19.0 \pm 2.2$ \\
$19251759+0053140$ & $111.4 \pm 3.2$ & $20.2 \pm 3.9$ & $7.2 \pm 1.4$ \\
$19261134+0051569$ & $125.2 \pm 11.1$ & $-92.3 \pm 13.9$ & $21.6 \pm 2.4$ \\
$19263808+0054441$ & $-32.2 \pm 0.4$ & $-41.4 \pm 0.8$ & $-21.2 \pm 2.4$ \\
$19264134+0137595$ & $98.6 \pm 6.0$ & $-31.5 \pm 7.1$ & $25.1 \pm 2.8$ \\
$19264917-0027469$ & - & - & - \\
$19265013+0149070$ & - & - & - \\
$19265193+0044004$ & $-15.8 \pm 1.6$ & $12.5 \pm 1.6$ & $-15.6 \pm 2.5$ \\
$19270600+0134446$ & - & - & - \\
$19270815+0017461$ & - & - & - \\
$19273856+0024149$ & - & - & - \\
$19274706+0023447$ & $117.2 \pm 9.1$ & $-54.6 \pm 11.3$ & $20.1 \pm 2.7$ \\
$19280508+0100139$ & $171.3 \pm 15.2$ & $-75.6 \pm 18.8$ & $18.3 \pm 3.3$ \\
$19283226+0033072$ & $102.3 \pm 5.0$ & $-34.9 \pm 6.5$ & $-5.2 \pm 0.9$ \\
\hline
\end{tabular}

data available for a subsample of our Li-rich giants. We also make use of the recent Gaia DR2 to compute luminosities and position the giants in the HR diagram. For the discussion in this section, we combine our sample of new discoveries with the Gaia-ESO Li-rich giants previously reported in Casey et al. (2016). For completeness, in the appendix we give both the observational data of these stars (Table B.1) and the results of the re-analysis of their spectra in Gaia-ESO iDR5 (Table B.2). Additional discussion of the Li-rich giants observed in the fields of open clusters is given in Appendix C. It is shown that these stars are not cluster members. An additional discussion of the giants observed towards the Bulge is given in Appendix D. It is also shown that Bulge membership is unlikely. 
A\&A 617, A4 (2018)

Table 8. Seismic estimates of the surface gravity and stellar masses of the Li-rich giants in the CoRoT fields.

\begin{tabular}{ccccccl}
\hline \hline CNAME & CoRoT ID & $\log g$ & $\sigma_{\log g}$ & $\begin{array}{c}\text { Mass } \\
M_{\odot}\end{array}$ & $\begin{array}{c}\sigma_{\text {mass }} \\
M_{\odot}\end{array}$ & $\begin{array}{l}\text { Num. } \\
\text { pipelines }\end{array}$ \\
\hline $19223053+0138518$ & 100440565 & 2.35 & 0.05 & 1.25 & 0.14 & 4 \\
$19251759+0053140$ & 100919702 & - & - & - & - & 0 \\
$19261134+0051569$ & 101064590 & 2.34 & 0.07 & 2.75 & 1.37 & 1 \\
$19263808+0054441$ & 101130864 & 2.48 & 0.02 & 0.91 & 0.12 & 2 \\
$19264134+0137595$ & 101139596 & 2.39 & 0.07 & 1.55 & 0.19 & 3 \\
$19264917-0027469$ & 101160340 & 1.93 & 0.06 & 1.46 & 0.46 & 1 \\
$19265013+0149070$ & 101162874 & - & - & - & - & 0 \\
$19265193+0044004$ & 101167637 & 2.42 & 0.03 & 1.20 & 0.15 & 4 \\
$19270600+0134446$ & 101205220 & 2.48 & 0.05 & 1.74 & 0.56 & 1 \\
$19270815+0017461$ & 101210895 & 2.23 & 0.02 & 1.29 & 0.18 & 1 \\
$19273856+0024149$ & 101292381 & 2.10 & 0.04 & 1.54 & 0.27 & 3 \\
$19274706+0023447$ & 101314825 & - & - & - & - & 0 \\
$19280508+0100139$ & 101351658 & 2.30 & 0.08 & 3.03 & 1.60 & 1 \\
$19283226+0033072$ & 101411079 & - & - & - & - & 0 \\
\hline
\end{tabular}

Notes. The error in $\log g$ takes into account the error in $T_{\text {eff }}$. Moreover, it includes both the standard and systematic errors for the targets for which detection was made by more than one pipeline. For the targets with only one determination, this is only the internal error of the pipeline (and thus it underestimates the uncertainty). The error in the masses also takes into account the error in $v_{\max }$, which is usually large. Moreover, we remark again that masses based on scaling relations need corrections that depend on the stellar parameters, as discussed in the text, and which were not applied here.

\subsection{Stellar properties from CoRoT data}

Perhaps the most important result based on the analysis of the CoRoT data ${ }^{1}$ of these new Li-rich giants is the evolutionary stage of star 19265193+0044004 (CoRoT 101167637). This star is a He-core burning clump giant according to Mosser et al. (2011). This is one of the few known Li-rich giant with a clear asteroseismic determination of the evolutionary stage, and the first based on CoRoT data. The other such giants include the clump giant reported by Silva Aguirre et al. (2014), the RGB bump giant reported by Jofré et al. (2015), and the two clump giants reported by Bharat Kumar et al. (2018), all with Kepler data. We also highlight the Li-rich giant found by Monaco et al. (2014) in one open cluster as its position at the CMD is consistent with the red clump. For the remaining Li-rich giants in our sample, the CoRoT data do not provide a clear evolutionary classification. In Sect.3.2, we estimated the giant to have 1.3-1.4 $\pm 0.3 M_{\odot}$. Thus, this is most likely a low-mass star, although the error bar does not exclude the possibility of an intermediate-mass value. As a low-mass star, it went through the He-core flash at the end of the RGB evolution. As suggested before (e.g. Kumar et al. 2011; Monaco et al. 2014), this episode must likely be related to the origin of the Li enrichment. Star 19265193+0044004 becomes now an important addition that supports the connection between Li-rich giants with the clump evolution.

Solar-like oscillations have been detected in the analysis of the light curves of 10 out of the 14 new Li-rich giants found in the CoRoT fields. Up to four pipelines were used in the seismic analysis (see Mosser \& Appourchaux 2009; Hekker et al. 2010; Mathur et al. 2010; de Assis Peralta et al. 2018). The frequency of maximum power, $v_{\max }$, together with our $T_{\text {eff }}$ values was used to compute seismic estimates of $\log g$ by means of a scaling relation (Brown et al. 1991; Kjeldsen \& Bedding 1995). Furthermore, using our temperatures, $v_{\max }$, and the large separation $(\Delta v)$, estimates of the stellar masses can also be obtained

\footnotetext{
1 The CoRoT data are publicly available and can be downloaded at http://idoc-corot.ias.u-psud.fr/
}

using scaling relations. See, for instance, Eq. (3) of Miglio et al. (2012). We used the following solar values: $T_{\text {eff } \odot}=5777 \mathrm{~K}$, $v_{\max \odot}=3090 \mu \mathrm{Hz}$, and $\Delta v_{\odot}=135 \mu \mathrm{Hz}$. The values are given in Table 8 .

Masses derived using global seismic parameters and scaling relations can be more accurate than those based on isochrones. However, the precision of the values obtained from the scaling relations itself depends on stellar parameters such as mass, metallicity, and evolutionary stage (e.g. Miglio et al. 2013). Corrections based on theoretical models and frequencies are required to improve the precision of stellar mass values, such as in Rodrigues et al. (2017) and Valentini et al. (in prep.). These corrections were not applied here. Therefore, the mass values should be seen only as indicative and used only as a check of the values derived previously using a different method. In most cases, the masses agree within the uncertainties with the values derived using UniDAM. In some cases of large disagreement, the seismic mass is based on one detection, hence this can be seen as a difficult and uncertain measurement.

The seismic $\log g$ values are mostly lower than the spectroscopic values. The mean difference is about $-0.16 \pm 0.13$ dex. For the Li-rich CoRoT giants of higher metallicity, this change moves some of the stars from inside the region of the RGB bump to a position around the clump or closer to the early AGB. The position of Li-rich CoRoT giants of lower metallicity is still consistent with the bump, although at higher stellar mass. Thus, the previous conclusion does not change. The stars remain consistent with three evolutionary stages: the RGB bump, the clump, and the early AGB.

Finally, De Medeiros et al. (2013) analysed the CoRoT light curve of $19273856+0024149$ and found it to display semisinusoidal variation, likely produced by rotation (we determined $v \sin i=12 \mathrm{~km} \mathrm{~s}^{-1}$ ). De Medeiros et al. (2013) derived a variability period of $74.383 \pm 1.0792$ days. These two measurements yield a "projected" radius of the star of $17.6 R_{\odot}$. Star $19273856+0024149$ is the giant in the right panel of Fig. 2 above the RGB bump above the blue track for $1.2 M_{\odot}$. The 


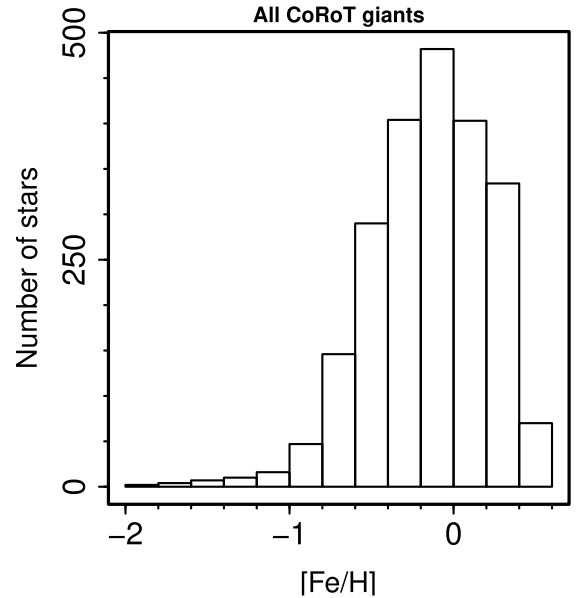

Fig. 5. Metallicity distribution of the CoRoT giants included in Gaia-ESO iDR5. Left: all giants in the CoRoT fields with determinations of Li abundances. Right: Li-rich giants. models for $1.2 M_{\odot}$ predict a radius of $\sim 11.6 R_{\odot}$ for its $\log g$ (2.39 dex, Table 2). A larger radius might mean that the giant is brighter and/or has higher mass than implied by our spectroscopic $\log g$ itself, bringing the star closer to the RGB bump. A higher mass value $\left(1.54 M_{\odot}\right)$ is supported by the seismic analysis.

\subsection{Giants in CoRoT fields}

The extended sample of CoRoT giants indicates that evolutionary stage is one main factor that unites Li-rich giants. Gaia-ESO has observed 2865 targets in CoRoT fields; 2431 of them have $\log g \leq 3.5$ in the iDR5 catalogue (and thus are considered here to be giants). Lithium abundances or upper limits were derived for 2252 of them.

The characteristics of the CoRoT data mean that the giants for which oscillations have been extracted are mostly in the range of the clump and bump, or somewhat lower on the RGB. These giants with asteroseismic data were the priority in the Gaia-ESO observations. Nevertheless, because of the difficulties of assigning fibres during the observations, these giants were not observed alone. The observed giants are distributed throughout the RGB, with $\log g$ ranging from 3.5 to 0.70 dex (with quartiles 2.74 and 2.30 dex). Within this large sample, we found a total of $23 \mathrm{Li}$-rich giants, including those reported in this work and in Casey et al. (2016). This is a fraction of $1.02 \%$, which is consistent with the numbers reported in the literature $(\sim 1-2 \%$, see e.g. Brown et al. 1989; Pilachowski et al. 2000; Kirby et al. 2016).

Histograms with the metallicity distribution of the CoRoT giants are shown in Fig. 5. The metallicity distribution of all the CoRoT giants spans from $[\mathrm{Fe} / \mathrm{H}]=-1.87$ to +0.52 , with mean $-0.15 \pm 0.36$ and quartiles at -0.38 and +0.13 . The Li-rich CoRoT giants have mean $[\mathrm{Fe} / \mathrm{H}]=-0.06 \pm 0.31$ and the quartiles of the distribution are at -0.34 and +0.21 . Thus, their metallicity distribution seems slightly shifted towards higher metallicities. The metallicity distribution is bimodal. There are two peaks, one at $[\mathrm{Fe} / \mathrm{H}]=-0.40(9$ stars below -0.20$)$ and another at +0.20 (11 stars above 0.0 ). Only two stars are found between $[\mathrm{Fe} / \mathrm{H}]=-0.20$ and 0.0 (and one more lacks determination of $[\mathrm{Fe} / \mathrm{H}])$. Given the small fraction of Li-rich giants, their numbers seems consistent with the metallicity distribution of the larger population.

The important result is that within this large sample of CoRoT giants, the Li-rich giants are mostly found in a narrow range of surface gravity values (i.e. narrow range of luminosities). These giants are mostly in the proximity of the RGB luminosity bump, although in particular for higher metallicity, some are also consistent with the position of the clump and/or could be at the early AGB. The asteroseismic data classify one star as a red clump giant, and at least one giant is visually consistent with the core He-burning stage at the intermediatemass regime, which might be even more challenging to explain. There is no extra-mixing event known to take place at this stage for intermediate-mass giants. The four exceptions likely have very uncertain atmospheric parameters.

The concentration around the three evolutionary regions is clearly visible in Fig. 6, even though the error bars prevent an accurate positioning of the objects. This observation differs from previous reports that Li-rich giants are located throughout the whole extension of the RGB. For example, Alcalá et al. (2011) reported 1 Li-rich low-mass M-type giant likely at the tip of the RGB. Monaco et al. (2011) discussed 5 Li-rich giants located between the RGB bump and the tip of the RGB. Martell \& Shetrone (2013) reported 23 Li-rich giants distributed from the bottom to the tip of the RGB.

This conclusion is also different from what has been reported in Casey et al. (2016), where most giants had been found to lie below the position of the RGB luminosity bump. This concentration seemed to suggest planet engulfment as the most likely scenario. We note here again that the new evolutionary tracks of Fu et al. (2018) argue for a lower position of the RGB bump of low-mass stars. For the sample of Casey et al. (2016), we used a new set of parameters revised during the Gaia-ESO iDR5 analysis. Most of the changes between the values of $T_{\text {eff }}$ and $\log g$ reported in Casey et al. (2016) and those reported here are well explained by the uncertainties in the measurements, however. Moreover, the seismic $\log g$ values place the stars closer to the red clump, making a position below the RGB bump even less likely.

Further motivation to review the likelihood of planet engulfment as a main channel behind the Li enrichment comes from recent works investigating $\mathrm{Be}$ abundances in Li-rich giants. Takeda \& Tajitsu (2017) attempted to detect Be in 20 Li-rich giants, including a few with $\mathrm{Li}$ abundances above the meteoritic value. No Be enhancement was detected. Adamów et al. (2018) recently reported an attempt to detect $\mathrm{Be}$ in two Li-rich giants. Again, the Be abundance was found to be depleted, as expected for red giants after the first dredge-up. Moreover, as discussed before, very fast rotation that clearly needs an additional mechanism to accelerate the stellar surface is seen in only two giants of our sample. The combination of all these observations seems 

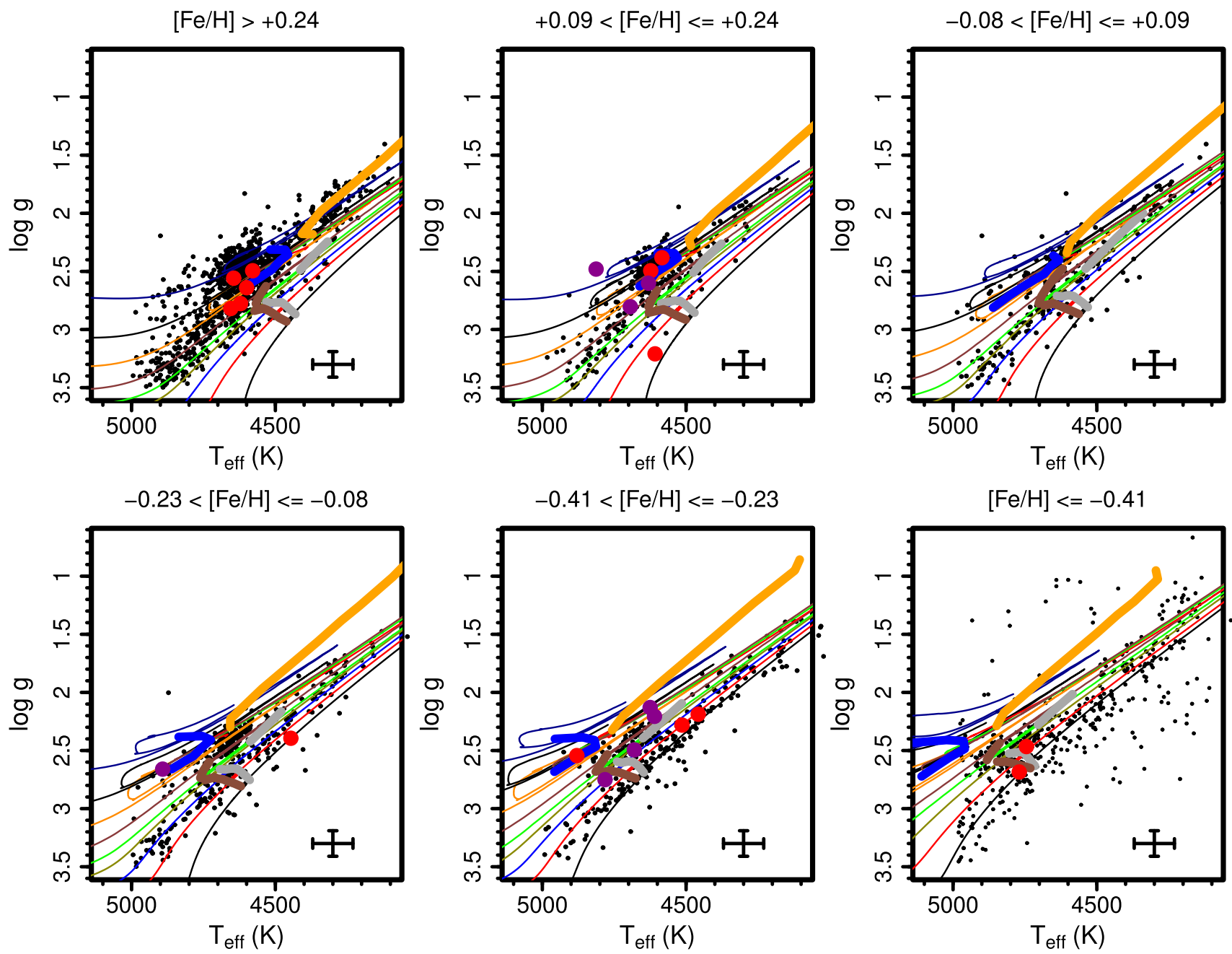

Fig. 6. All CoRoT giants with detected Li abundances or upper limits in the iDR5 catalogue. The giants found to be Li-rich are highlighted. The tracks are as those shown in Fig. 2. The red symbols are the new discoveries reported in this paper, while the dark magenta symbols are the Li-rich giants reported by Casey et al. (2016).

to suggest that planet engulfment plays a minor role at most in the formation process of Li-rich giants.

\subsection{Luminosities with Gaia DR2 data}

As mentioned before, the Gaia DR2 parallaxes and proper motions are given in Table A.1. To calculate stellar luminosities, we assumed that the stellar distance is the inverse of the parallax. This assumption should provide accurate results for most of our stars, but not for all of them. We refer to Luri et al. (2018) for a discussion of the uncertainties, correlations, and limitations of the parallaxes. We computed luminosities for the 27 giants whose relative uncertainty of the parallaxes is lower than $15 \%$.

To compute the absolute magnitudes, $K s$ from 2MASS was transformed into $K$ in the CIT/CTIO system (Elias et al. 1982) using the relation from Carpenter (2001) and ignoring the reddening. Bolometric corrections in $K$ have been tabulated by Houdashelt et al. (2000). The bolometric correction mostly depends on the $T_{\text {eff }}$ of the giant, is only weakly dependent on the metallicity, and is mostly independent of $\log g$. Thus, in the grid of Houdashelt et al. (2000), we first selected the table of closer metallicity (either $[\mathrm{Fe} / \mathrm{H}]=0.00$ or $-0.50 \mathrm{dex}$ ) and linearly interpolated the values in $T_{\text {eff }}$. Failing to interpolate in $[\mathrm{Fe} / \mathrm{H}]$ causes an effect of at most 0.02 mag in the bolometric correction. This effect is negligible given that the uncertainties in our $T_{\text {eff }}$ values can cause an effect of about $0.2 \mathrm{mag}$ in the bolometric correction. Luminosities were computed using a solar bolometric magnitude of $M_{\mathrm{bol} \odot}=4.75 \mathrm{mag}$.

We estimated the uncertainties in the luminosities coming from the uncertainties in the parallaxes, $K s$ magnitudes, and bolometric corrections. To do this, we assumed that the observed value is the mean of a Gaussian distribution with a standard deviation equal to the observed error (assumed to be $0.2 \mathrm{mag}$ in the case of the bolometric correction). We then repeated the calculation of the luminosities 10000 times by drawing a random value of parallax, magnitude, and bolometric correction out of these distributions. The standard deviation of the resulting distribution of luminosity values was taken to be the uncertainty in this quantity. The median of the uncertainties in the luminosities is 0.12 dex.

The position in the HR diagram of the Li-rich giants for which we computed luminosities is shown in Fig. 7. The plot is divided into metallicity bins as in Fig. 2 and shows the same evolutionary tracks. It is clear that the HR diagram shows a similar behaviour as the $T_{\text {eff }}-\log g$ diagram. The low-mass giants are concentrated around the position of the luminosity bump or the clump. The intermediate-mass stars seem to be at the core-He 

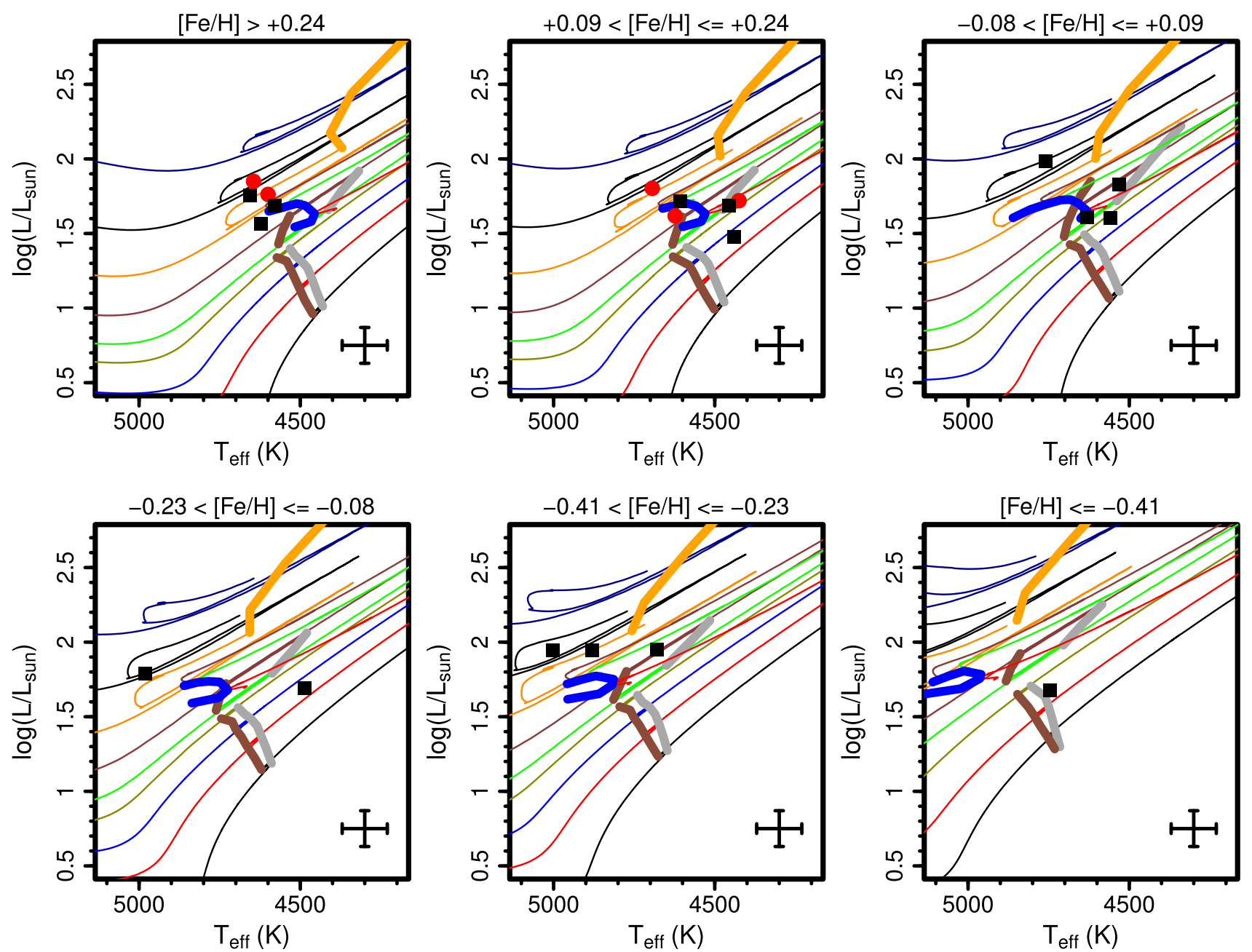

Fig. 7. HR diagram with $21 \mathrm{Li}$-rich giants discovered by the Gaia-ESO Survey that have good values of Gaia parallaxes, divided according to metallicity into different panels. The tracks are the same as in Fig. 2. The range of $[\mathrm{Fe} / \mathrm{H}]$ is given at the top of each panel. Super Li-rich giants with $A(\mathrm{Li})>3.3$ dex (in non-LTE) are shown as red circles, and giants with a Li abundance below this are shown as black squares. Typical error bars, $\pm 70 \mathrm{~K}$ in $T_{\text {eff }}$ and \pm 0.12 in $\log \left(L / L_{\odot}\right)$, are shown in the bottom right corners of the panels.

burning stage. A few stars might be at the early AGB, given the uncertainties.

Luminosity values of higher quality may be obtained with a better estimation of the distances and by taking into account the reddening. We note, however, that a reddening of 1 mag would increase the luminosity by $0.4 \mathrm{dex}$. This change in the luminosities would not change our conclusions. The giants would still be located around the bump, the core-He burning regions, or the early AGB. Thus, the Gaia data seem to confirm our previous conclusions.

As a final comment, we note that star $18265248+0627259$, which seemed to be a core-He burning intermediate-mass star in Fig. 2, is in the same region in Fig. 7 (bottom left panel). This position makes internal mixing of fresh Li unlikely in this star. This fast rotator is also one of the candidates for planet engulfment. A follow-up investigation of its Be abundance would be an interesting way to search for additional support for this hypothesis.

\section{Summary}

We reported on the discovery of 20 new Li-rich giants observed by the Gaia-ESO Survey. Four giants were observed in the field of open clusters, but do not seem to be members. Two giants were observed in fields towards the Galactic bulge, but magnitudes and proper motions are not compatible with bulge membership. The remaining 14 giants were observed in the CoRoT fields.

The asteroseismic data classify star $19265193+0044004$ as a He-core burning clump giant. To the best of our knowledge, this is only the fifth $\mathrm{Li}$-rich giant with asteroseismic determination of the evolutionary stage; it is the first with CoRoT data. It becomes the fourth such star to be found at the red clump. Its evolutionary stage supports a possible connection between the He-flash and the surface Li enrichment.

A comprehensive investigation of additional properties (IR magnitudes, rotational velocities, strong lines, and additional chemical abundances) did not reveal common peculiarities shared by all the Li-rich giants. We were able to identify one star with enhanced Ba abundance (19265013+0149070), five giants with $v \sin i>10 \mathrm{~km} \mathrm{~s}^{-1}$, and one star with IR excess (19270600+0134446). All giants show disc-like motion. Eleven stars seem to belong to the thin disc, and seven have enhanced $[\alpha / \mathrm{Fe}]$, which tentatively classifies them as thick-disc stars.

The two fastest rotators in our sample are candidates for having suffered planet engulfment. Otherwise, the only common 
characteristic of the Li-rich giants in our sample seems to be their evolutionary stage. The Li-rich giants are mostly located around three evolutionary stages: the RGB luminosity bump, the clump, and the early AGB.

The concentration around these three evolutionary stages is particularly clear in the sample of giants in the CoRoT fields observed within the Gaia-ESO Survey. Lithium abundances (or upper limits) are available for 2252 such giants, covering from the bottom to the upper regions of the RGB (for low-mass stars) and up to the early AGB of intermediate-mass stars. In this extended sample, the $1 \%$ of the giants that were found to be Li-rich are only located around the RGB luminosity bump, the clump, or the early AGB. Luminosities computed using Gaia DR2 parallaxes also support these conclusions.

This observation suggests that evolutionary stage plays a major role in the process of $\mathrm{Li}$ enrichment, at least in this sample. Additional processes such as planet engulfment probably only play a minor role. However, Li-rich objects found in other evolutionary stages cannot be explained in the same way. This includes the Li-rich metal-poor dwarfs and subgiants that were found, for example, in the globular cluster NGC 6397 by Koch et al. (2011), in M 30 by Gruyters et al. (2016), and in the field by Li et al. (2018). Deep mixing that would add freshly synthesised $\mathrm{Li}$ is not possible in these stars. Thus, they likely require external pollution. Even in these cases, however, the nondetection of Be enhancement in the Li-rich dwarf in NGC 6397 argues against planet engulfment (Pasquini et al. 2014).

Acknowledgements. We thank the referee for the quick reports and for the useful suggestions that helped to improve the clarity of the paper. R. Smiljanic acknowledges support from the Polish Ministry of Science and Higher Education. G. Tautvaišienè acknowledges support from the European Social Fund via the Lithuanian Science Council grant No. 09.3.3-LMT-K-712-01-0103. V.A. and S.G.S acknowledge the support from Fundação para a Ciência e a Tecnologia (FCT, Portugal) through the research grant through national funds and by FEDER through COMPETE2020 by grants UID/FIS/04434/2013 \& POCI-01-0145-FEDER-007672, PTDC/FIS-AST/1526/2014 \& POCI-01-0145-FEDER-016886 and PTDC/FIS AST/7073/2014 \& POCI-01-0145-FEDER-016880. V.A. and S.G.S also acknowledge support from FCT through Investigador FCT contracts nr. IF/00650/2015/CP1273/CT0001 and IF/00028/2014/CP1215/CT0002. T.B. was funded by the project grant "The New Milky Way" from the Knut and Alice Wallenberg Foundation. JM acknowledges support from the ERC Consolidator Grant funding scheme (project STARKEY, G.A. n. 615604). T. Morel acknowledges financial support from Belspo for contract PRODEX GAIADPAC and PLATO. T. Masseron acknowledges support provided by the Spanish Ministry of Economy and Competitiveness (MINECO) under grant AYA 2017-88254-P. R.A.G acknowledges the support of the PLATO/CNES grant S.M. acknowledges support from NASA grant NNX15AF13G and NSF grant AST-1411685 and the Ramon y Cajal fellowship no. RYC-2015-17697. AB acknowledges support from the Millennium Science Initiative (Chilean Ministry of Economy). The research leading to the presented results has received funding from the European Research Council under the European Community's Seventh Framework Programme (FP7/2007-2013)/ERC grant agreement no 338251 (StellarAges). Based on data products from observations made with ESO Telescopes at the La Silla Paranal Observatory under programme ID 188.B-3002. These data products have been processed by the Cambridge Astronomy Survey Unit (CASU) at the Institute of Astronomy, University of Cambridge, and by the FLAMES/UVES reduction team at INAF/Osservatorio Astrofisico di Arcetri. These data have been obtained from the Gaia-ESO Survey Data Archive, prepared and hosted by the Wide Field Astronomy Unit, Institute for Astronomy, University of Edinburgh, which is funded by the UK Science and Technology Facilities Council. This work was partly supported by the European Union FP7 programme through ERC grant number 320360 and by the Leverhulme Trus through grant RPG-2012-541. We acknowledge the support from INAF and Ministero dell' Istruzione, dell' Università' e della Ricerca (MIUR) in the form of the grant "Premiale VLT 2012". The results presented here benefit from discussions held during the Gaia-ESO workshops and conferences supported by the ESF (European Science Foundation) through the GREAT Research Network Programme. This research has made use of: NASA's Astrophysics Data System; the SIMBAD database, operated at CDS, Strasbourg, France; the VizieR catalogue access tool, CDS, Strasbourg, France. The original description of the VizieR service was published in Ochsenbein et al. (2000); data products from the Wide-field Infrared Survey Explorer, which is a joint project of the University of California, Los Angeles, and the Jet Propulsion Laboratory/California Institute of Technology, funded by the National Aeronautics and Space Administration; data products from the Two Micron All Sky Survey, which is a joint project of the University of Massachusetts and the Infrared Processing and Analysis Center/California Institute of Technology, funded by the National Aeronautics and Space Administration and the National Science Foundation; the WEBDA database, operated at the Department of Theoretical Physics and Astrophysics of the Masaryk University. The analysis has made extensive use of R (R Core Team 2016), RStudio (RStudio Team 2015), and the R packages FITSio (Harris 2013), gplots (Warnes et al. 2015), and stringr (Wickham 2015). This work has made use of data from the European Space Agency (ESA) mission Gaia (https: //www . cosmos. esa.int/gaia), processed by the Gaia Data Processing and Analysis Consortium (DPAC, https://www.cosmos.esa.int/web/ gaia/dpac/consortium). Funding for the DPAC has been provided by national institutions, in particular the institutions participating in the Gaia Multilateral Agreement.

\section{References}

Adamów, M., Niedzielski, A., Kowalik, K., et al. 2018, A\&A, 613, A47 Adibekyan, V. Z., Santos, N. C., Sousa, S. G., \& Israelian, G. 2011, A\&A, 535, L11

Aguilera-Gómez, C., Chanamé, J., Pinsonneault, M. H., \& Carlberg, J. K. 2016, ApJ, 829, 127

Alcalá, J. M., Biazzo, K., Covino, E., Frasca, A., \& Bedin, L. R. 2011, A\&A, $531, \mathrm{~L} 12$

Alonso-Santiago, J., Negueruela, I., Marco, A., et al. 2017, MNRAS, 469, 1330 Alves-Brito, A., Meléndez, J., Asplund, M., Ramírez, I., \& Yong, D. 2010, A\&A, 513, A35

Anthony-Twarog, B. J., Deliyannis, C. P., Rich, E., \& Twarog, B. A. 2013, ApJ, 767, L19

Ashwell, J. F., Jeffries, R. D., Smalley, B., et al. 2005, MNRAS, 363, L81

Auvergne, M., Bodin, P., Boisnard, L., et al. 2009, A\&A, 506, 411

Baglin, A., Auvergne, M., Boisnard, L., et al. 2006, in 36th COSPAR Scientific Assembly, COSPAR Meeting, 36

Bedding, T. R., Mosser, B., Huber, D., et al. 2011, Nature, 471, 608

Bharat Kumar, Y., Reddy, B. E., Muthumariappan, C., \& Zhao, G. 2015, A\&A, 577, A10

Bharat Kumar, Y., Singh, R., Eswar Reddy, B., \& Zhao, G. 2018, ApJ, 858, L22

Böcek Topcu, G., Afşar, M., Schaeuble, M., \& Sneden, C. 2015, MNRAS, 446, 3562

Böcek Topcu, G., Afşar, M., \& Sneden, C. 2016, MNRAS, 463, 580

Borucki, W. J., Koch, D., Basri, G., et al. 2010, Science, 327, 977

Bressan, A., Marigo, P., Girardi, L., et al. 2012, MNRAS, 427, 127

Brown, J. A., Sneden, C., Lambert, D. L., \& Dutchover, E. 1989, ApJS, 71, 293 Brown, T. M., Gilliland, R. L., Noyes, R. W., \& Ramsey, L. W. 1991, ApJ, 368, 599

Cameron, A. G. W., \& Fowler, W. A. 1971, ApJ, 164, 111

Carlberg, J. K., Majewski, S. R., \& Arras, P. 2009, ApJ, 700, 832

Carlberg, J. K., Smith, V. V., Cunha, K., et al. 2015, ApJ, 802, 7

Carlberg, J. K., Cunha, K., \& Smith, V. V. 2016a, ApJ, 827, 129

Carlberg, J. K., Smith, V. V., Cunha, K., \& Carpenter, K. G. 2016b, ApJ, 818, 25 Carpenter, J. M. 2001, AJ, 121, 2851

Casey, A. R., Ruchti, G., Masseron, T., et al. 2016, MNRAS, 461, 3336

Cassisi, S., Salaris, M., \& Pietrinferni, A. 2016, A\&A, 585, A124

Castilho, B. V., Gregorio-Hetem, J., Spite, F., Spite, M., \& Barbuy, B. 1998, A\&AS, 127, 139

Castilho, B. V., Spite, F., Barbuy, B., et al. 1999, A\&A, 345, 249

Charbonnel, C., \& Balachandran, S. C. 2000, A\&A, 359, 563

Cutri, R. M., et al. 2012, VizieR Online Data Catalog: II/311

Cutri, R. M., et al. 2013, VizieR Online Data Catalog: II/328

de Assis Peralta, R., Samadi, R., \& Michel, E. 2018, Astron. Nachr., 339, 134

de Castro, D. B., Pereira, C. B., Roig, F., et al. 2016, MNRAS, 459, 4299

de la Reza, R., Drake, N. A., \& da Silva, L. 1996, ApJ, 456, L115

de la Reza, R., Drake, N. A., da Silva, L., Torres, C. A. O., \& Martin, E. L. 1997, ApJ, 482, L77

de la Reza, R., Drake, N. A., Oliveira, I., \& Rengaswamy, S. 2015, ApJ, 806, 86

de Medeiros, J. R., Lebre, A., de Garcia Maia, M. R., \& Monier, R. 1997, A\&A, 321, L37

De Medeiros, J. R., Ferreira Lopes, C. E., Leão, I. C., et al. 2013, A\&A, 555, A63

Dehnen, W., \& Binney, J. J. 1998, MNRAS, 298, 387 
Dekker, H., D’Odorico, S., Kaufer, A., Delabre, B., \& Kotzlowski, H. 2000, in Optical and IR Telescope Instrumentation and Detectors, eds. M. Iye, \& A. F. Moorwood, SPIE Conf. Ser., 4008, 534

Delgado Mena, E., Tsantaki, M., Sousa, S. G., et al. 2016, A\&A, 587, A66

Drake, N. A., de la Reza, R., da Silva, L., \& Lambert, D. L. 2002, AJ, 123, 2703

Drazdauskas, A., Tautvaišienè, G., Randich, S., et al. 2016a, A\&A, 589, A50

Drazdauskas, A., Tautvaišienè, G., Smiljanic, R., Bagdonas, V., \& Chorniy, Y 2016b, MNRAS, 462, 794

Elias, J. H., Frogel, J. A., Matthews, K., \& Neugebauer, G. 1982, AJ, 87, 1029

Elsworth, Y., Hekker, S., Basu, S., \& Davies, G. R. 2017, MNRAS, 466, 3344

Escorza, A., Boffin, H. M. J., Jorissen, A., et al. 2017, A\&A, 608, A100

Frasca, A., Biazzo, K., Alcalá, J. M., et al. 2017, A\&A, 602, A33

Frasca, A., Guillout, P., Klutsch, A., et al. 2018, A\&A, 612, A96

Fu, X., Bressan, A., Marigo, P., et al. 2018, MNRAS, 476, 496

Gaia Collaboration (Prusti, T., et al.) 2016, A\&A, 595, A1

Gaia Collaboration (Brown, A. G. A., et al.) 2018, A\&A 616, A1

Gilmore, G., Randich, S., Asplund, M., et al. 2012, The Messenger, 147, 25

Gregorio-Hetem, J., Castilho, B. V., \& Barbuy, B. 1993, A\&A, 268, L25

Grevesse, N., \& Sauval, A. J. 1998, Space Sci. Rev., 85, 161

Grevesse, N., Asplund, M., \& Sauval, A. J. 2007, Space Sci. Rev., 130, 105

Gruyters, P., Lind, K., Richard, O., et al. 2016, A\&A, 589, A61

Gustafsson, B., Edvardsson, B., Eriksson, K., et al. 2008, A\&A, 486, 951

Handberg, R., Brogaard, K., Miglio, A., et al. 2017, MNRAS, 472, 979

Harris, A. 2013, FITSio: FITS (Flexible Image Transport System) utilities, R package version 2.0-0

Heiter, U., Lind, K., Asplund, M., et al. 2015, Phys. Scr., 90, 054010

Hekker, S., Broomhall, A.-M., Chaplin, W. J., et al. 2010, MNRAS, 402, 2049

Henden, A. A., Levine, S., Terrell, D., \& Welch, D. L. 2015, in AAS Meet. Abstr., $225,336.16$

Houdashelt, M. L., Bell, R. A., \& Sweigart, A. V. 2000, AJ, 119, 1448

Jackson, R. J., Jeffries, R. D., Lewis, J., et al. 2015, A\&A, 580, A75

Jasniewicz, G., Parthasarathy, M., de Laverny, P., \& Thévenin, F. 1999, A\&A, 342,831

Jeffries, R. D. 1997, MNRAS, 292, 177

Jofré, E., Petrucci, R., García, L., \& Gómez, M. 2015, A\&A, 584, L3

Johnson, D. R. H., \& Soderblom, D. R. 1987, AJ, 93, 864

Kővári, Z., Korhonen, H., Strassmeier, K. G., et al. 2013, A\&A, 551, A2

Kővári, Z., Strassmeier, K. G., Carroll, T. A., et al. 2017, A\&A, 606, A42

Kirby, E. N., Guhathakurta, P., Zhang, A. J., et al. 2016, ApJ, 819, 135

Kjeldsen, H., \& Bedding, T. R. 1995, A\&A, 293, 87

Koch, A., Lind, K., \& Rich, R. M. 2011, ApJ, 738, L29

Kriskovics, L., Kővári, Z., Vida, K., Granzer, T., \& Oláh, K. 2014, A\&A, 571, A74

Kumar, Y. B., Reddy, B. E., \& Lambert, D. L. 2011, ApJ, 730, L12

Kurucz, R. 1993, ATLAS9 Stellar Atmosphere Programs and 2 km/s grid, CD ROM No. 13. (Cambridge, MA: Smithsonian Astrophysical Observatory), 13

Lagarde, N., Decressin, T., Charbonnel, C., et al. 2012, A\&A, 543, A108

Lambert, D. L., Smith, V. V., \& Heath, J. 1993, PASP, 105, 568

Lanzafame, A. C., Frasca, A., Damiani, F., et al. 2015, A\&A, 576, A80

Li, H., Aoki, W., Matsuno, T., et al. 2018, ApJ, 852, L31

Lind, K., Asplund, M., \& Barklem, P. S. 2009, A\&A, 503, 541

Lindegren, L., Hernandez, J., Bombrun, A., et al. 2018, A\&A, 616, A2

Luri, X., Brown, A. G. A., Sarro, L. M., et al. 2018, A\&A, 616, A9

Lyubimkov, L. S. 2016, Astrophysics, 59, 411

Magrini, L., Randich, S., Kordopatis, G., et al. 2017, A\&A, 603, A2

Magrini, L., Spina, L., Randich, S., et al. 2018, A\&A, in press, DOI: 10.1051/0004-6361/201832841

Martell, S. L., \& Shetrone, M. D. 2013, MNRAS, 430, 611

Mathur, S., García, R. A., Régulo, C., et al. 2010, A\&A, 511, A46

Melo, C. H. F., de Laverny, P., Santos, N. C., et al. 2005, A\&A, 439, 227

Merle, T., Van Eck, S., Jorissen, A., et al. 2017, A\&A, 608, A95

Miglio, A., Brogaard, K., Stello, D., et al. 2012, MNRAS, 419, 2077

Miglio, A., Chiappini, C., Morel, T., et al. 2013, Eur. Phys. J. Web Conf., 43, 03004

Mikolaitis, Š., Hill, V., Recio-Blanco, A., et al. 2014, A\&A, 572, A33

Minniti, D., Lucas, P., \& VVV Team. 2017, VizieR Online Data Catalog: II/348

Mints, A., \& Hekker, S. 2017, A\&A, 604, A108

Monaco, L., Villanova, S., Moni Bidin, C., et al. 2011, A\&A, 529, A90

Monaco, L., Boffin, H. M. J., Bonifacio, P., et al. 2014, A\&A, 564, L6

Mosser, B., \& Appourchaux, T. 2009, A\&A, 508, 877

Mosser, B., Barban, C., Montalbán, J., et al. 2011, A\&A, 532, A86

Nataf, D. M., Gould, A., Fouqué, P., et al. 2013, ApJ, 769, 88

Ochsenbein, F., Bauer, P., \& Marcout, J. 2000, A\&AS, 143, 23
Palacios, A., Charbonnel, C., \& Forestini, M. 2001, A\&A, 375, L9

Palmerini, S., Cristallo, S., Busso, M., et al. 2011, ApJ, 741, 26

Pancino, E., Lardo, C., Altavilla, G., et al. 2017, A\&A, 598, A5

Pasquini, L., Avila, G., Blecha, A., et al. 2002, The Messenger, 110, 1

Pasquini, L., Koch, A., Smiljanic, R., Bonifacio, P., \& Modigliani, A. 2014, A\&A, 563, A3

Pilachowski, C. A., Sneden, C., Kraft, R. P., Harmer, D., \& Willmarth, D. 2000, AJ, 119, 2895

Privitera, G., Meynet, G., Eggenberger, P., et al. 2016a, A\&A, 591, A45

Privitera, G., Meynet, G., Eggenberger, P., et al. 2016b, A\&A, 593, A128

R Core Team 2016, R: A Language and Environment for Statistical Computing, (Vienna, Austria: R Foundation for Statistical Computing)

Randich, S., \& Gilmore, G. 2013, The Messenger, 154, 47

Rebull, L. M., Carlberg, J. K., Gibbs, J. C., et al. 2015, AJ, 150, 123

Recio-Blanco, A., de Laverny, P., Kordopatis, G., et al. 2014, A\&A, 567, A5

Reddy, A. B. S., \& Lambert, D. L. 2016, A\&A, 589, A57

Rodrigues, T. S., Bossini, D., Miglio, A., et al. 2017, MNRAS, 467, 1433

RStudio Team 2015, RStudio: Integrated Development Environment for R, (Boston, MA: RStudio, Inc.)

Sacco, G. G., Morbidelli, L., Franciosini, E., et al. 2014, A\&A, 565, A113

Sackmann, I.-J., \& Boothroyd, A. I. 1999, ApJ, 510, 217

Saito, R. K., Hempel, M., Minniti, D., et al. 2012a, A\&A, 537, A107

Saito, R. K., Minniti, D., Dias, B., et al. 2012b, A\&A, 544, A147

Scalo, J. M., Despain, K. H., \& Ulrich, R. K. 1975, ApJ, 196, 805

Siess, L., \& Livio, M. 1999, MNRAS, 308, 1133

Silva Aguirre, V., Ruchti, G. R., Hekker, S., et al. 2014, ApJ, 784, L16

Skrutskie, M. F., Cutri, R. M., Stiening, R., et al. 2006, AJ, 131, 1163

Smiljanic, R., Korn, A. J., Bergemann, M., et al. 2014, A\&A, 570, A122

Smiljanic, R., Franciosini, E., Randich, S., et al. 2016, A\&A, 591, A62

Spina, L., Randich, S., Magrini, L., et al. 2017, A\&A, 601, A70

Stello, D., Meibom, S., Gilliland, R. L., et al. 2011, ApJ, 739, 13

Sumi, T. 2004, MNRAS, 349, 193

Szigeti, L., Mészáros, S., Smith, V. V., et al. 2018, MNRAS, 474, 4810

Takeda, Y., \& Tajitsu, A. 2017, PASJ, 69, 74

Tautvaišienè, G., Drazdauskas, A., Mikolaitis, Š., et al. 2015, A\&A, 573, A55

Tautvaišienė, G., Drazdauskas, A., Bragaglia, A., Randich, S., \& Ženovienė, R. 2016, A\&A, 595, A16

Teixeira, R., Galli, P. A. B., Benevides-Soares, P., et al. 2011, A\&A, 534, A91

Udalski, A., Szymanski, M., Kubiak, M., et al. 2002, Acta Astron., 52, 217

Vieira, K., Casetti-Dinescu, D. I., Méndez, R. A., et al. 2007, AJ, 134, 1432

Wallerstein, G., \& Sneden, C. 1982, ApJ, 255, 577

Warnes, G. R., Bolker, B., Bonebakker, L., et al. 2015, gplots: Various R Programming Tools for Plotting Data, $\mathrm{r}$ package version 2.17.0

Wickham, H. 2015, stringr: Simple, Consistent Wrappers for Common String Operations, R package version 1.0.0

Wright, E. L., Eisenhardt, P. R. M., Mainzer, A. K., et al. 2010, AJ, 140, 1868

Zacharias, N., Monet, D. G., Levine, S. E., et al. 2004, AAS Meet. Abstr., 36, 1418

1 Nicolaus Copernicus Astronomical Center, Polish Academy of Sciences, Bartycka 18, 00-716 Warsaw, Poland

e-mail: rsmiljanic@camk .edu.pl

2 INAF - Osservatorio Astrofisico di Arcetri, Largo E. Fermi 5, 50125, Florence, Italy

3 INAF - Osservatorio di Astrofisica e Scienza dello Spazio di Bologna, via Gobetti 93/3, 40129 Bologna, Italy

4 Institute of Theoretical Physics and Astronomy, Vilnius University, Saulètekio av. 3, 10257 Vilnius, Lithuania

5 Dipartimento di Fisica \& Astronomia, Università degli Studi di Bologna, via Gobetti 93/2, 40129 Bologna, Italy

6 Space Science Data Center - Agenzia Spaziale Italiana, via del Politecnico, s.n.c., 00133 Roma, Italy

7 Instituto de Astrofísica e Ciências do Espaço, Universidade do Porto, CAUP, Rua das Estrelas, 4150-762 Porto, Portugal

8 Dipartimento di Fisica e Astronomia Galileo Galilei, Università di Padova, Vicolo dell'Osservatorio 3, 35122 Padova, Italy

9 European Southern Observatory, Karl-Schwarzschild-Strasse 2, 85748 Garching bei München, Germany

10 IRFU, CEA, Université Paris-Saclay, 91191 Gif-sur-Yvette, France

11 Université Paris Diderot, AIM, Sorbonne Paris Cité, CEA, CNRS, 91191 Gif-sur-Yvette, France 
12 Instituto de Astrofísica de Canarias, 38205 La Laguna, Tenerife, Spain

13 Universidad de La Laguna, Dept. Astrofísica, 38206 La Laguna, Tenerife, Spain

14 Space Science Institute, 4750 Walnut Street, Suite 205, Boulder, CO 80301, USA

15 LESIA, Observatoire de Paris, Université PSL, CNRS, Sorbonne Université, Université Paris Diderot, Sorbonne Paris Cité, 92195 Meudon, France

16 Max-Planck-Institut for Solar System Research, Justus-von-LiebigWeg 3, 37077 Göttingen, Germany

17 Stellar Astrophysics Centre, Department of Physics and Astronomy, Aarhus University, Ny Munkegade 120, 8000 Aarhus C, Denmark

18 Max-Planck Institut für Astronomie, Königstuhl 17, 69117 Heidelberg, Germany

19 Leibniz-Institut für Astrophysik Potsdam (AIP), An der Sternwarte 16, 14482 Potsdam, Germany

20 Space Sciences, Technologies and Astrophysics Research (STAR) Institute, Université de Liège, Quartier Agora, Allée du 6 Août 19c, Bât. B5C, 4000-Liège, Belgium

21 School of Physics, University of New South Wales, Sydney, NSW 2052, Australia

22 Institute of Astronomy, University of Cambridge, Madingley Road, Cambridge CB3 OHA, UK

23 Lund Observatory, Department of Astronomy and Theoretical Physics, Box 43, 22100 Lund, Sweden
${ }^{24}$ INAF - Padova Observatory, Vicolo dell'Osservatorio 5, 35122 Padova, Italy

25 Department of Physics and Astronomy, Uppsala University, Box 516, 75120 Uppsala, Sweden

26 Dipartimento di Fisica e Astronomia, Sezione Astrofisica, Universitá di Catania, via S. Sofia 78, 95123 Catania, Italy

27 Laboratoire Lagrange (UMR7293), Université de Nice Sophia Antipolis, CNRS, Observatoire de la Côte d'Azur, CS 34229, 06304 Nice Cedex 4, France

28 Instituto de Física y Astronomía, Universidad de Valparaíso, Chile

29 Departamento de Didáctica, Universidad de Cádiz, 11519 Puerto Real, Cádiz, Spain

30 INAF - Osservatorio Astrofisico di Catania, via S. Sofia 78, 95123 Catania, Italy

31 Núcleo de Astronomía, Facultad de Ingeniería, Universidad Diego Portales, Av. Ejército 441, Santiago, Chile

32 Laboratoire d'astrophysique, Ecole Polytechnique Fèdèrale de Lausanne (EPFL), Observatoire de Sauverny, 1290 Versoix, Switzerland

33 Departamento de Ciencias Fisicas, Universidad Andres Bello, Fernandez Concha 700, Las Condes, Santiago, Chile

34 INAF - Osservatorio Astronomico di Palermo, Piazza del Parlamento 1, 90134 Palermo, Italy

35 European Southern Observatory, Alonso de Cordova 3107 Vitacura, Santiago de Chile, Chile

36 Núcleo Milenio Formación Planetaria - NPF, Universidad de Valparaíso, Av. Gran Bretaña 1111, Valparaíso, Chile 
R. Smiljanic et al.: Properties of newly discovered Li-rich giants

\section{Appendix A: Gaia DR2 data for the Li-rich giants discovered in the Gaia-ESO Survey}

Table A.1. Gaia DR2 photometric and astrometric information for the complete list of 40 Li-rich giants discovered by the Gaia-ESO Survey.

\begin{tabular}{|c|c|c|c|c|c|c|}
\hline CNAME & Gaia DR2 Designation & $\begin{array}{c}G \\
\mathrm{mag}\end{array}$ & $\begin{array}{c}\pi \\
\text { mas }\end{array}$ & $\begin{array}{c}\text { pmRA } \\
\text { mas } y^{-1}\end{array}$ & $\begin{array}{l}\text { pmDec } \\
\text { mas yr }^{-1}\end{array}$ & $\log \left(L / L_{\odot}\right)$ \\
\hline $08405045-5508509$ & 10495901509520192 & & $4 \pm 0.01$ & & & $9 \pm 0.08$ \\
\hline $17522490-2927512$ & 56552974204511104 & 3 & $8 \pm 0.07$ & $-1.77 \pm($ & \pm 0.09 & - \\
\hline $17531013-2932063$ & 4056548065148248576 & 13.71 & $0.36 \pm 0.05$ & $+1.00 \pm 0.07$ & $+0.78 \pm 0.05$ & - \\
\hline $18181062-3246291$ & 4045590259161801088 & 11.69 & $0.73 \pm 0.05$ & $+7.33 \pm 0.08$ & $+0.69 \pm 0.07$ & $1.60 \pm 0.11$ \\
\hline $18182698-3242584$ & 4045596512634175232 & & & $-2.41 \pm 0.08$ & $-6.64 \pm 0.07$ & $1.72 \pm 0.13$ \\
\hline $18265248+0627259$ & 77215166550061184 & 14.04 & $0.26 \pm 0.03$ & $+1.47 \pm 0.05$ & $-6.58 \pm 0.05$ & $1.79 \pm 0.13$ \\
\hline $5=2 \cdot 012$ & 6848 & & & & .08 & $1.69 \pm 0.10$ \\
\hline $19251759+0053140$ & 4263760067953345920 & 14.02 & $0.40 \pm 0.04$ & $-2.75 \pm 0.07$ & $-3.28 \pm 0.06$ & $1.56 \pm 0.11$ \\
\hline $19261134+0051569$ & 4287730555301937664 & & $0.25 \pm 0.03$ & $-4.53 \pm 0.05$ & $-6.57 \pm 0.05$ & $1.68 \pm 0.14$ \\
\hline 192 & 48 & 12.61 & $0.59 \pm 0.04$ & $+3.38 \pm$ & -3 & $1.75 \pm 0.10$ \\
\hline & 56 & 13.81 & & -3.6 & -3 & $1.85 \pm 0.13$ \\
\hline 469 & 50379904 & 15.49 & $0.22 \pm 0.05$ & $-3.79 \pm 0.08$ & $-6.22 \pm 0.06$ & - \\
\hline 070 & 25536128 & 15.28 & $0.16 \pm$ & $-3.57 \pm$ & \pm 0.06 & - \\
\hline 192 & 400 & 12.58 & 0.4 & +2.8 & +0 & $1.94 \pm 0.12$ \\
\hline & & & & -1.3 & 08 & - \\
\hline & 60 & & & $-4.21 \pm$ & -6. & - \\
\hline 24149 & 5984 & 14.80 & $0.14 \pm$ & $-2.77 \pm$ & \pm 0.05 & - \\
\hline 192 & 864 & 44 & 0.2 & -4.01 & \pm 0.05 & $1.72 \pm 0.14$ \\
\hline & & & & -4.4 & -6 & $1.62 \pm 0.15$ \\
\hline 072 & 06048 & 14.21 & $0.27 \pm$ & -2.0 & \pm 0.04 & $1.76 \pm 0.12$ \\
\hline $08095783-4701385$ & 80241807744 & 12.27 & $0.44 \pm 0.03$ & $-10.11 \pm 0.07$ & $+5.06 \pm 0.06$ & $1.95 \pm 0.10$ \\
\hline $116-4740125$ & 707586560 & 13.74 & $0.22 \pm 0.02$ & $-4.27 \pm 0.03$ & $+4.86 \pm 0.04$ & $2.07 \pm 0.11$ \\
\hline & 21606400 & & & -3.1 & \pm 0.04 & $1.98 \pm 0.15$ \\
\hline & 472 & 14.92 & & -6.1 & +6 & $1.83 \pm 0.14$ \\
\hline $10300194-6321203$ & 5252183088177166208 & 13.63 & $0.35 \pm 0.02$ & $-9.74 \pm 0.03$ & $+3.79 \pm 0.02$ & $1.67 \pm 0.09$ \\
\hline $10323205-6324012$ & 5251997786110150016 & 13.31 & $0.44 \pm 0.01$ & $-12.34 \pm 0.03$ & $+3.82 \pm 0.02$ & $1.48 \pm 0.08$ \\
\hline & & & $0.42 \pm 0$ & $-4.22 \pm 0.02$ & $+2.15 \pm 0.02$ & $1.69 \pm 0.09$ \\
\hline 10 & 216 & 13.93 & $0.24 \pm 0$ & $-10.53 \pm$ & \pm 0.03 & $1.93 \pm 0.11$ \\
\hline $15-7623259$ & 666727680 & 12.73 & $0.53 \pm 0.02$ & $-11.65 \pm 0.04$ & $+3.50 \pm 0.03$ & $1.89 \pm 0.09$ \\
\hline $18033785-3009201$ & 607210512512 & 13.64 & $0.35 \pm 0.03$ & $+0.36 \pm 0.07$ & $-2.10 \pm 0.05$ & $1.69 \pm 0.11$ \\
\hline $35+0123293$ & 358460629632 & 15.34 & & $-5.28 \pm 0.06$ & $-5.82 \pm 0.06$ & - \\
\hline $19242472+0044106$ & 4263 & 13.78 & $0.42 \pm 0.03$ & $-4.20 \pm 0.06$ & $-7.83 \pm 0.05$ & $1.61 \pm 0.11$ \\
\hline $19252571+0031444$ & 4263697189630154112 & 14.94 & $0.18 \pm 0.04$ & $-1.91 \pm 0.09$ & $-3.88 \pm 0.07$ & - \\
\hline $19252758+0153065$ & 4288618754548267904 & 13.36 & $0.33 \pm 0.03$ & $-2.44 \pm 0.06$ & $-3.08 \pm 0.05$ & $1.80 \pm 0$ \\
\hline $19252837+0027037$ & 4263682689819998208 & 15.83 & $0.09 \pm 0.05$ & $-3.67 \pm 0.08$ & $-3.61 \pm 0.07$ & - \\
\hline $19253819+0031094$ & 60162048 & 15.25 & $0.16 \pm$ & $-2.89 \pm$ & $-7.22 \pm 0.07$ & - \\
\hline 19261007-0010200 & 3514379648 & & $0.22 \pm 0.02$ & $-3.38 \pm 0$ & $-2.06 \pm 0.04$ & $1.95 \pm 0.12$ \\
\hline $19264038-0019575$ & 4263381629788846720 & 14.94 & $0.23 \pm 0.04$ & $-3.32 \pm 0.06$ & $-9.02 \pm 0.05$ & - \\
\hline 19301883-0004175 & 4215367778069753728 & 14.13 & $0.15 \pm 0.04$ & $-3.82 \pm 0.06$ & $-5.68 \pm 0.05$ & - \\
\hline $19304281+2016107$ & 2017726986620705152 & 14.53 & $0.25 \pm 0.04$ & $-0.89 \pm 0.04$ & $-5.39 \pm 0.05$ & $1.99 \pm 0.15$ \\
\hline
\end{tabular}

Notes. The luminosities computed as discussed in the text are given in the last column. The new discoveries are listed in the top part of the table, and the stars from Casey et al. (2016) appear in the bottom part. 
Appendix B: Tables with data for the Li-rich giants reported in Casey et al. (2016)

Table B.1. Observational data of the Li-rich giants reported in Casey et al. (2016).

\begin{tabular}{lcccccrr}
\hline \hline CNAME & Field & 2MASS ID & $\begin{array}{c}\text { RA } \\
\text { h:m:s (J2000) }\end{array}$ & $\begin{array}{c}\text { Dec } \\
\text { d:m:s (J2000) }\end{array}$ & $\begin{array}{c}V \\
\text { mag }\end{array}$ & $\begin{array}{c}\text { RV } \\
\mathrm{km} \mathrm{s}^{-1}\end{array}$ & $S / N$ \\
\hline $08095783-4701385$ & $\gamma^{2}$ Vel & $08095784-4701383$ & $08: 09: 57.83$ & $-47: 01: 38.5$ & 10.82 & +26.1 & 145 \\
$08102116-4740125$ & $\gamma^{2}$ Vel & $08102116-4740125$ & $08: 10: 21.16$ & $-47: 40: 12.5$ & $14.22^{1}$ & +70.7 & 129 \\
$08110403-4852137$ & NGC 2547 & $08110403-4852137$ & $08: 11: 04.03$ & $-48: 52: 13.7$ & 14.87 & +54.2 & 58 \\
$08395152-5315159$ & IC 2391 & $08395152-5315159$ & $08: 39: 51.52$ & $-53: 15: 15.9$ & 15.41 & +27.0 & 102 \\
$10300194-6321203$ & IC 2602 & $10300194-6321203$ & $10: 30: 01.94$ & $-63: 21: 20.3$ & 14.16 & -10.4 & 145 \\
$10323205-6324012$ & IC 2602 & $10323205-6324012$ & $10: 32: 32.05$ & $-63: 24: 01.2$ & 13.72 & +13.3 & 88 \\
$10495719-6341212$ & IC 2602 & $10495719-6341212$ & $10: 49: 57.19$ & $-63: 41: 21.2$ & $13.84^{1}$ & +13.8 & 123 \\
$10503631-6512237$ & IC 2602 & $10503632-6512237$ & $10: 50: 36.31$ & $-65: 12: 23.7$ & 12.77 & -34.1 & 84 \\
$11000515-7623259$ & Cha I & $11000515-7623259$ & $11: 00: 05.15$ & $-76: 23: 25.9$ & $13.74^{1}$ & -15.8 & 103 \\
$18033785-3009201$ & Bulge & $18033785-3009200$ & $18: 03: 37.85$ & $-30: 09: 20.1$ & $13.27^{1}$ & -69.9 & 97 \\
$19230935+0123293$ & Corot & $19230934+0123293$ & $19: 23: 09.35$ & $+01: 23: 29.3$ & 15.93 & +11.9 & 7 \\
$19242472+0044106$ & Corot & $19242474+0044104$ & $19: 24: 24.73$ & $+00: 44: 10.5$ & 14.45 & +77.7 & 92 \\
$19252571+0031444$ & Corot & $19252571+0031444$ & $19: 25: 25.71$ & $+00: 31: 44.4$ & $15.10^{1}$ & -38.5 & 44 \\
$19252758+0153065$ & Corot & $19252758+0153064$ & $19: 25: 27.58$ & $+01: 53: 06.5$ & 13.73 & +28.1 & 35 \\
$19252837+0027037$ & Corot & $19252837+0027037$ & $19: 25: 28.37$ & $+00: 27: 03.7$ & $16.02^{1}$ & +0.1 & 28 \\
$19253819+0031094$ & Corot & $19253819+0031094$ & $19: 25: 38.19$ & $+00: 31: 09.4$ & 15.59 & +26.4 & 33 \\
$19261007-0010200$ & Corot & $19261020+0010226$ & $19: 26: 10.07$ & $+00: 10: 20.0$ & $14.55^{1}$ & -21.1 & 63 \\
$19264038-0019575$ & Corot & - & $19: 26: 40.38$ & $+00: 19: 57.5$ & - & +42.3 & 21 \\
$19301883-0004175$ & Corot & - & $19: 30: 18.83$ & $+00: 04: 17.5$ & - & +57.3 & 41 \\
$19304281+2016107$ & NGC 6802 & $19304281+2016107$ & $19: 30: 42.81$ & $+20: 16: 10.7$ & $14.67^{3}$ & +17.4 & 67 \\
\hline
\end{tabular}

Notes. The $V$ magnitudes are from APASS (the AAVSO Photometric All-Sky Survey, Henden et al. 2015) unless otherwise noted: (1) NOMAD catalogue (Zacharias et al. 2004); (2) The Guide Star Catalog, Version 2.3.2 (GSC2.3) (STScI, 2006).

Table B.2. New iDR5 atmospheric parameters and lithium abundances for the Li-rich giants reported in Casey et al. (2016).

\begin{tabular}{lccccccccccc}
\hline \hline CNAME & $\begin{array}{c}T_{\text {eff }} \\
(\mathrm{K})\end{array}$ & $\begin{array}{c}\sigma \\
(\mathrm{K})\end{array}$ & $\log g$ & $\sigma$ & {$[\mathrm{Fe} / \mathrm{H}]$} & $\sigma$ & $\begin{array}{c}\xi \\
\mathrm{km} \mathrm{s}^{-1}\end{array}$ & $\begin{array}{c}\sigma \\
\mathrm{km} \mathrm{s}^{-1}\end{array}$ & $\begin{array}{c}A(\mathrm{Li}) \\
(\mathrm{LTE})\end{array}$ & $\begin{array}{c}\sigma(\mathrm{Li}) \\
\text { (non-LTE) }\end{array}$ \\
\hline $08095783-4701385$ & 5002 & 28 & 2.55 & 0.04 & -0.25 & 0.02 & 1.50 & 0.00 & 3.60 & - & 3.24 \\
$08102116-4740125$ & 4433 & 175 & - & - & -0.12 & 0.01 & - & - & 3.52 & 0.11 & - \\
$08110403-4852137$ & 4599 & 212 & - & - & -0.07 & 0.08 & - & - & $3.60^{1}$ & 0.13 & - \\
$08395152-5315159$ & 4531 & 187 & 2.52 & 0.18 & +0.01 & 0.05 & - & - & $1.88^{1}$ & 0.29 & 2.07 \\
$10300194-6321203$ & 4472 & 184 & - & - & -0.03 & 0.04 & - & - & $2.89^{1}$ & 0.28 & - \\
$10323205-6324012$ & 4440 & 178 & 2.57 & 0.19 & +0.15 & 0.01 & - & - & $2.96^{1}$ & 0.23 & 2.89 \\
$10495719-6341212$ & 4646 & 117 & - & - & +0.02 & 0.09 & - & - & $2.97^{1}$ & 0.20 & - \\
$10503631-6512237$ & 4580 & 119 & - & - & -0.03 & 0.05 & - & - & 2.50 & 0.17 & - \\
$11000515-7623259$ & 4418 & - & - & - & +0.14 & 0.00 & - & - & 2.55 & 0.07 & - \\
$18033785-3009201$ & 4455 & 58 & 2.43 & 0.11 & +0.12 & 0.07 & 1.64 & 0.18 & 2.66 & 0.13 & 2.67 \\
$19230935+0123293$ & 4610 & 144 & 2.21 & 0.21 & -0.33 & 0.55 & - & - & 2.59 & 0.15 & 2.57 \\
$19242472+0044106$ & 4631 & 58 & 2.60 & 0.12 & +0.09 & 0.06 & 1.73 & 0.20 & 2.51 & 0.06 & 2.57 \\
$19252571+0031444$ & 4892 & 169 & 2.66 & 0.25 & -0.17 & 0.20 & - & - & 2.32 & 0.11 & 2.38 \\
$19252758+0153065$ & 4694 & 46 & 2.80 & 0.10 & +0.22 & 0.26 & - & - & 3.54 & 0.07 & 3.35 \\
$19252837+0027037$ & 4813 & 236 & 2.48 & 0.19 & +0.14 & 0.24 & - & - & 3.32 & 0.13 & 3.17 \\
$19253819+0031094$ & 4625 & 247 & 2.13 & 0.41 & -0.40 & 0.27 & - & - & 3.28 & 0.07 & 3.06 \\
$19261007-0010200$ & 4680 & 35 & 2.49 & 0.09 & -0.35 & 0.18 & - & - & 3.26 & 0.06 & 3.02 \\
$19264038-0019575$ & 4782 & 46 & 2.75 & 0.10 & -0.40 & 0.28 & - & - & 3.69 & 0.16 & 3.34 \\
$19301883-0004175$ & 4128 & 77 & 1.22 & 0.31 & - & - & - & - & 2.14 & 0.17 & 2.13 \\
$19304281+2016107$ & 4759 & 67 & 2.63 & 0.12 & -0.04 & 0.11 & 1.80 & 0.09 & 2.62 & 0.06 & 2.63 \\
\hline
\end{tabular}

Notes. (1) The values of Li abundance for these five stars are not part of the final iDR5 Gaia-ESO catalogue. The values are missing from the main catalogue, likely because abundance measurements performed by different pipelines disagreed by a large margin. We report here instead the $\mathrm{Li}$ abundances rederived by only one of these pipelines, that of the Arcetri node (see description of this analysis node in Lanzafame et al. 2015). 


\section{Appendix C: Giants in open cluster fields}

We have identified four Li-rich giants in the field of three open clusters. If the stars are indeed members of the clusters, we could use the known cluster distances and reddening values to accurately position the objects in a CMD. This would allow a more robust understanding of their evolutionary stage than is possible with the spectroscopic diagram of Fig. 2 (see, e.g. the clump giant found by Monaco et al. 2014, in the open cluster Trumpler 5). Often, however, radial velocities and/or metallicities indicate that the Li-rich giants seem to be just field stars that are unrelated to the cluster (see Alonso-Santiago et al. 2017; Frasca et al. 2017, for recent examples).

The four giants reported here do not seem to be cluster members. This was also the case of the Li-rich giants in open cluster fields found by Casey et al. (2016).

Membership is excluded based on discrepant RVs. Star CNAME 08405643-5308309 has an RV $=+55 \mathrm{~km} \mathrm{~s}^{-1}$, while the open cluster IC 2391 has a mean RV $15 \mathrm{~km} \mathrm{~s}^{-1}$ (based on five stars reported in Spina et al. 2017). Stars CNAME 175224902927512 and 17531013-2932063 have +81.6 and $-25.8 \mathrm{~km} \mathrm{~s}^{-1}$, respectively, while the cluster Rup 134 has a mean RV $\sim-41 \mathrm{~km} \mathrm{~s}^{-1}$ (Magrini et al. 2018). Star CNAME 18265248+ 0627259 (the fastest rotator in the sample) has an $\mathrm{RV}=$ $+32.7 \mathrm{~km} \mathrm{~s}^{-1}$, while NGC 6633 has a mean RV -29 $\mathrm{km} \mathrm{s}^{-1}$ (Magrini et al. 2017).

Six giants from Casey et al. (2016), all observed in the field of open clusters, are missing $\log g$ values in the iDR5 catalogue (Table B.2). This happened because the disagreement between the two pipelines deriving $\log g$ values for these stars increased in the new analysis cycle (the pipelines are described in Lanzafame et al. 2015). These values were thus considered unreliable and discarded during the homogenisation stage. To have an indicative value of $\log g$, we retrieved the values of one of these pipelines (the one that remained more consistent between the different analysis cycles). The $\log g$ values for these giants are between 2.56 and 2.78 . The $T_{\text {eff }}$ values are between 4400 and $4650 \mathrm{~K}$ (Table B.2). We verified that these values place the giants exactly around the RGB bump of low-mass stars in the right panel of Fig. 2. Thus, they would still support the conclusion drawn from the remaining stars.

\section{Appendix D: Giants towards the bulge}

Casey et al. (2016) reported the discovery of one Li-rich giant towards the bulge (CNAME 18033785-3009200), which seemed to have properties (RV and abundances) consistent with bulge membership. Here, we report on two additional Li-rich giants observed in fields towards the bulge (CNAME 181810623246291 and 18182698-3242584).

Similarly as with open clusters, if we can confirm their membership to the bulge, we could use the known bulge distance and reddening maps (e.g. Nataf et al. 2013) to accurately position the objects in a CMD.

One of our two new Li-rich giants, 18182698-3242584, has the highest Li enrichment discovered so far in the Gaia-ESO Survey, with $A(\mathrm{Li})=4.04$ in non-LTE.

The two new giants have been included in a few proper motion studies dedicated to bulge fields (Vieira et al. 2007; Teixeira et al. 2011). In particular, Vieira et al. (2007) discussed the distribution of proper motions of bulge stars at Plaut's low extinction window; $(l, b)=\left(0^{\circ}, 8^{\circ}\right)$. In this field, the distributions of proper motions of bulge stars in Galactic coordi-

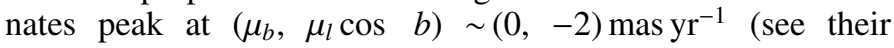
Fig. 8). The proper-motion dispersion of the bulge stars is found to be $(3.39 \pm 0.11,2.91 \pm 0.09)$. The proper motions derived by Vieira et al. (2007) for stars 18181062-3246291 and $18182698-3242584$ are $\left(\mu_{b}, \mu_{l} \cos b\right)=(6.8 \pm 0.7,-5.9 \pm 0.7)$ and $(-7.5 \pm 0.04,-1.5 \pm 0.3)$, respectively. The values are only marginally consistent with the typical bulge values, suggesting that the giants are probably not bulge stars.

The two new Li-rich giants are part of fields observed by the VVV survey (Vista Variables in the Vía Láctea, Saito et al. 2012a; Minniti et al. 2017), but when measurements are given, all magnitudes are flagged as unreliable (probably because of saturation). The unreliable $K s$ value for $18181062-3246291$ is $9.21 \mathrm{mag}$, which would be much brighter than the typical $K s$ magnitude of bulge clump giants in the same field, $K s \sim 12.7-$ 13.4 (Saito et al. 2012b).

Star 18033785-3009200, reported in Casey et al. (2016), has been observed by OGLE (Optical Gravitational Lensing Experiment, Udalski et al. 2002) and has magnitudes $I=12.63$ mag and $V=14.18 \mathrm{mag}$. Its VVV $K s=11.07 \mathrm{mag}$ is this time more reliable. In both cases, the magnitudes again seem to be too bright for bulge giants (see, e.g. Sumi 2004; Nataf et al. 2013).

Moreover, the distance we derived using UniDAM for star 18181062-3246291 and the distances based on Gaia parallaxes are too small to be consistent with bulge membership. We thus conclude that most likely none of the three Gaia-ESO Li-rich giants observed in bulge fields belongs to the bulge itself. 